\title{
Recent advancements in metal organic framework based electrodes for supercapacitors
}

\author{
Yujie Zhao ${ }^{1}$, Jinzhang $\mathrm{Liu}^{1^{*}}$, Michael Horn ${ }^{2}$, Nunzio Motta ${ }^{2}$, Mingjun $\mathrm{Hu}^{1}$ and Yan $\mathrm{Li}^{1^{*}}$
}

\begin{abstract}
Metal organic frameworks (MOFs) are considered as very promising candidates to build electrodes for electrochemical energy storage devices such as lithium ion batteries, fuel cells and supercapacitors, due to their diverse structure, adjustable aperture, large specific surface area and abundant active sites. Supercapacitor has been widely investigated in the past decades. Of critical importance in these devices is the electrode active materials, and this application has been intensively studied with the development of novel nanomaterials. In this review we summarize recent reports on MOFs as electrode materials for supercapacitors. Specifically, the synthesis of MOF materials for supercapacitor electrodes and their performance in electrochemical energy storage are discussed. We aim to include supercapacitor electrode materials related to MOFs, such as carbon, metal and composite materials. It is proposed that MOFs play an important role in the development of a new generation of supercapacitor electrode materials. Finally, we discuss the current challenges in the field of supercapacitors, with a view towards how to address these challenges with the future development of MOFs and their derivatives.
\end{abstract}

Keywords: metal organic frameworks (MOFs), electrochemistry, supercapacitors, electrode, derivative

\section{INTRODUCTION}

Supercapacitors, taking advantage of the properties of conventional capacitors and ionic batteries, have attracted widespread research focus in the past four decades. Their unique properties, including high power density, fast charge/discharge rate, long cycle life and environmental friendliness, make supercapacitors attractive devices for a variety of applications in energy storage such as electronic communications, the emergent electric transportation industry and aerospace [1-10].

The energy storage mechanism of supercapacitors can be generally classified into two categories: electric doublelayer capacitance (EDLC) and pseudocapacitance [1,1114]. EDLC is based on the physical adsorption and desorption of ions at the interface between an electrolyte and electrode. Normally, carbon-based materials (activated carbon, graphene, carbon nanotubes, etc.) with good conductivity and high specific surface area are used as electrode active materials [15,16]. Pseudocapacitance stores charge via reversible redox reactions between an electrode and electrolyte. Electrode active materials can be metal oxides $\left(\mathrm{NiO}_{x}, \mathrm{RuO}_{2}, \mathrm{MnO}_{2}\right.$, etc. $)$ or conductive polymers (polyaniline, polypyrrole, etc.) [17-20]. In general, the specific capacitance of these materials is higher than that of the carbon-based materials in EDLC supercapacitor electrodes, owing to the chemical adsorption and desorption of $\mathrm{H}^{+}$in acidic electrolyte or alkali metal cations in alkaline media [21-25]. However, there is debate on the classification of pseudocapacitive materials, because some exhibit distinct redox peaks in cyclic voltammetry $(\mathrm{CV})$ loops as ionic battery materials do. In most cases, the capacitance of pseudocapacitive nanostructures is contributed by both Faradaic and EDLC. The EDLC contribution becomes more dominant with reducing the size, i.e., increasing the specific surface area, of pseudocapacitive nanocrystals.

In order to develop high-performance active materials for supercapacitor electrodes, several key aspects are focused on. Desirable characteristics in these materials include adjustable pore size distribution, high accessible specific surface area (SSA), high electrochemical activity and good electrical conductivity [26-28]. Carbon-based materials are superior in terms of SSA, electrical con-

${ }^{1}$ School of Materials Science and Engineering, Beihang University, Beijing 100191, China

${ }^{2}$ School of Chemistry, Physics, and Mechanical Engineering, Queensland University of Technology, Brisbane 4001, QLD, Australia

"Corresponding authors (emails: ljz78@buaa.edu.cn (Liu J); liyan@buaa.edu.cn (Li Y)) 
ductivity and chemical stability, but along with relative lower specific capacitance and energy density $[4,16,29]$. For pseudocapacitive materials, relatively lower surface area and inferior porosity suppress the dissociation of active component and ions from electrolyte and slow the electron or ion transfer, resulting in a limited power density. Furthermore, the redox reaction of the electrode material cannot be fully completed in the rapid charge and discharge process, which leads to a lower rate capability $[22,23]$. The development of new materials for improved supercapacitor electrodes requires maintaining the above desirable characteristics, while addressing the limitations of currently used materials.

Metal organic frameworks (MOFs) are a new category of porous materials consisting of metal ions or ion clusters and organic ligands connected through coordination bonds, auxiliary hydrogen bonds, metallic bonding, or van der Waals force interaction. It is also known as "coordination polymer" formed via self-assembly, resulting in a highly structured infinite network [30-33]. When compared with traditional porous materials, MOFs have the advantages of diverse scaffold structure, adjustable pore size, large SSA, and an abundance of active sites. Currently, MOFs have been widely used in gas adsorption and separation, catalysis, drug delivery, imaging, and sensors $[31,34,35]$. Interest in this new type of porous material is growing rapidly as its potential application in many fields is becoming realized. In recent years, MOFs and their derivatives have also been applied in the field of electrochemical energy storage, such as in lithium-ion batteries, fuel cells and supercapacitors. However, since MOFs are crystalline due to their ordered network constructed through coordination bonds between ligands and the metal center, the skeleton of MOFs is flexible and the electrical conductivity is generally inferior to carbonaceous electrode materials. Consequently, MOFs and MOF derivatives with good stability and high conductivity are highly urgent in the development of supercapacitor electrodes [36-42].

This paper reviews the recent research progress of MOFs and their derivatives in the application of supercapacitors. The content is divided into three sections. (1) MOFs applied in supercapacitors. (2) MOF derivatives including porous carbons, metal oxides and metal sulfides that maintain the characteristics of MOFs, and are used to make supercapacitor electrodes. (3) MOF composite electrodes. This section discusses the composite materials based on MOFs or their derivatives for supercapacitor electrodes. Finally, we summarize the current problems of MOFs and their derivatives in the field of supercapacitor electrodes as well as their possible direction for development in the future.

\section{MOFS DIRECTLY USED AS SUPERCAPACITOR ELECTRODES}

MOFs show strong potential for advanced supercapacitor electrodes due to their inherent properties including large SSA, adjustable pore size and abundance of active sites [43]. The large SSA of MOFs can increase the contact area between MOFs electrode and electrolyte, which further improves the specific capacitance. Furthermore, the adjustable pore size can ensure the fast transport of electrons and electrolyte ions while the abundant active sites can promote the pseudocapacitance. The specific capacitance was calculated by CV and galvanostatic charge/ discharge (GCD). The areal capacitance is used to represent the capacitance per square centimetre apparent area of activated material. What is more, improving the specific capacitance of electrode materials is an effective way to get high energy density because the energy density $(E)$ of supercapacitor is proportional to the specific capacitance $(C)$ according to the equation [44]: $E=C V^{2} / 2$, where $E$ is the energy, $C$ is the capacitance, and $V$ is the voltage. Apart from the capacitance, widening voltage window is the other approach to improve the energy density, while it is related to the type of electrolyte.

Lee et al. [45] synthesised Co-based MOFs at $100^{\circ} \mathrm{C}$ using cobalt nitrate and terephtalic acid for supercapacitors. A Co-MOF film with a uniform thickness of $5 \mu \mathrm{m}$ was constructed by MOF nanoparticles ranging from 50 to $100 \mathrm{~nm}$ (Fig. 1a). The Co-MOF film delivered a maximum pseudocapacitance of $206.76 \mathrm{~F} \mathrm{~g}^{-1}$ in $\mathrm{LiOH}$ electrolyte at $0.6 \mathrm{~A} \mathrm{~g}^{-1}$ (Fig. 1c), together with strong cycling stability, retaining $98.5 \%$ of the initial capacity after 1000 cycles. This is attributed to the fine crystalline structure of the MOF, stable in various electrolytes, as evidenced by XRD (Fig. 1b). In $\mathrm{LiOH}$ electrolyte, the organic ligands of the MOF can be continuously separated and recombined with the central metal ions, resulting in a high cyclic stability for Co-MOF films. The stability of Co-MOFs is on a par with Co-oxide materials $[46,47]$. It was noteworthy that the change of the highly crystalline phase of $\mathrm{CoO}$ was observed in $\mathrm{LiCl}$ and $\mathrm{KCl}$ electrolytes. Among Co-based oxides, only $\mathrm{Co}_{3} \mathrm{O}_{4}$ has good capacitance properties, so the appearance of $\mathrm{CoO}$ leads to lower capacitance in $\mathrm{LiCl}$ and $\mathrm{KCl}$ electrolyte (Fig. 1c).

Following the above report, Lee et al. [48] further studied the effects of organic linkers with different molecular lengths on producing Co-MOFs with different 

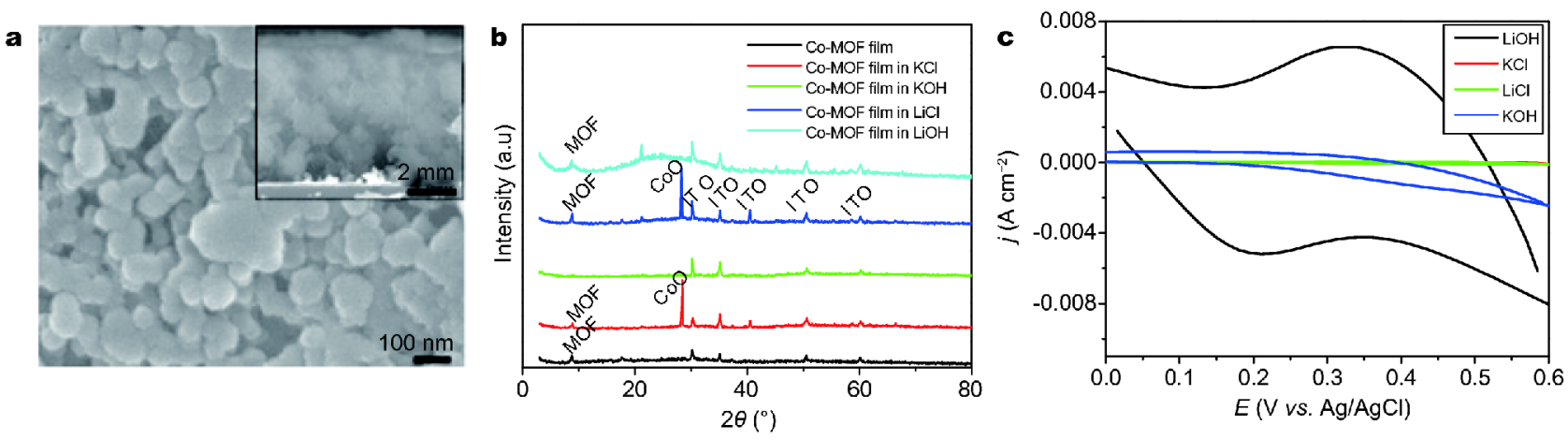

Figure 1 (a) Scanning electron microscopy (SEM) top and cross-sectional view (inset) of Co-MOF films; (b) X-ray diffraction (XRD) patterns of CoMOF films after 1000 cycles in different electrolytes; (c) CV curves of Co-MOF film in different electrolytes. Reproduced with permission from Ref. [45], Copyright 2012, Elsevier.
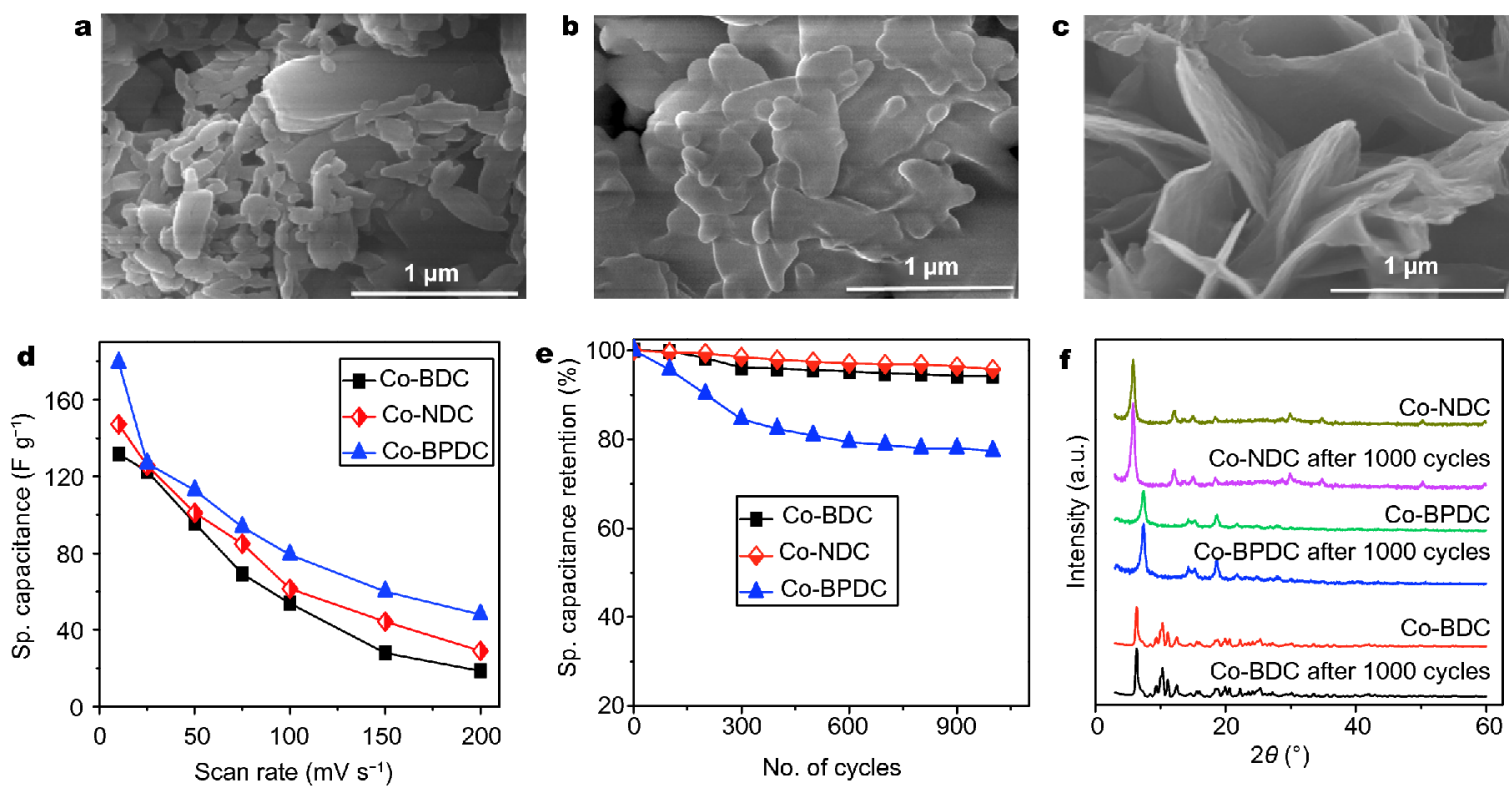

Figure 2 SEM images of (a) Co-BDC, (b) Co-NDC and (c) Co-BPDC MOF films on an ITO-glass substrate. (d) Specific capacitance of different CoMOFs at various scan rates; (e) cycllic stability of different Co-MOFs at $100 \mathrm{mV} \mathrm{s}^{-1}$; (f) XRD patterns of different Co-MOFs after 1000 cycles at $100 \mathrm{mV} \mathrm{s}^{-1}$. Reproduced with permission from Ref. [48], Copyright 2013, Elsevier.

pore sizes and surface areas (Fig. 2a-c). Typically, three organic linkers, 1,4-benzenedicarboxylic acid $\left(\mathrm{H}_{2} \mathrm{BDC}\right)$, 2,6-naphthalenedicarboxylc $\left(\mathrm{H}_{2} \mathrm{NPC}\right)$ and 4,4-biphenyldicarboxylic $\left(\mathrm{H}_{2} \mathrm{BPDC}\right)$ with increased molecular lengths were used. These Co-MOF films were produced using blade coating technique as previously employed by Lee et al. [45]. They found that a longer organic linker produced larger pores and larger surface area, compared with a shorter linker. The best performance was obtained from the MOF employing the longest linker, $\mathrm{H}_{2} \mathrm{BPDC}$. This material was denoted as Co-BPDC and possessed a pore mode distribution about $78.96 \mathrm{~nm}$, and an associated SSA of $138.35 \mathrm{~m}^{2} \mathrm{~g}^{-1}$. The Co-BPDC displayed a maximum capacitive performance of $179.2 \mathrm{~F} \mathrm{~g}^{-1}$ (Fig. 2d) at a scanning rate of $10 \mathrm{mV} \mathrm{s}^{-1}$. In addition, the loss of capacitance was $6 \%$ after 1000 cycles for these Co-BPDC based electrodes (Fig. 2e), and the structure was not obviously altered (Fig. 2f). These results indicate that the larger effective surface area and pore diameter have a positive effect on the capacitance of the supercapacitors constructed by these MOFs.

Miles et al. [49] also prepared cobalt based MOFs. The electrically insulating Co-MOF-71 1,4-benzenedicarboxylate in $\mathrm{N}, \mathrm{N}$-dimethylformamide [Co(BDC)DMF)] was obtained by reacting a cobalt salt with the organic ligand $\mathrm{H}_{2} \mathrm{BDC}$. This work demonstrated how this 3-D co- 


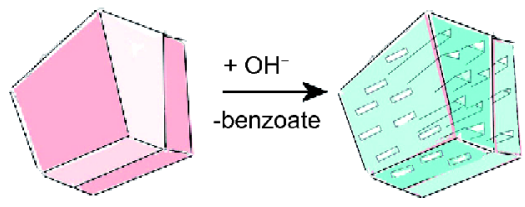

Figure 3 Schematic of a "conformal transformation" with formation of a porous product. Reproduced with permission from Ref. [49], Copyright 2013, Elsevier.

ordination polymer may be induced to become electrochemically active via "conformal transformation", whereby the chemical structure is altered without significant modification of the crystalline shape. $\mathrm{Co}^{2+}$ acts as the central metal ion reacting with $\mathrm{OH}^{-}$to produce $\mathrm{Co}(\mathrm{OH})_{2}$ in a strong alkaline environment, and the product possesses a more highly porous structure, suitable for psuedocapacitance (Fig. 3). In this report the CoMOF-71 was able to be fully transformed in alkaline electrolytes and displayed modest specific capacitances at $\mathrm{pH} \geq 11$.

Ni-based MOFs also attract great interest due to their unique structure as well as Co-MOFs reviewed above. Yang et al. [50] reported a high performance Ni-MOF-24 prepared by nickel chloride and $p$-benzodicarboxylic acid (PTA). The capacitance of Ni-MOF-24 was up to $1,127 \mathrm{~F} \mathrm{~g}^{-1}$ in $6 \mathrm{~mol} \mathrm{~L}^{-1} \mathrm{KOH}$ electrolyte at the current density of $0.5 \mathrm{~A} \mathrm{~g} \mathrm{~g}^{-1}$. Meanwhile, a good cyclic stability of over $90 \%$ capacitance retention was obtained after 3,000 cycles. The excellent performance was ascribed to the layered structure and favorable exposed facets of the asprepared Ni-based MOF material.

Yan et al. [51] prepared Ni-MOFs (Fig. 4) with a novel accordion morphology using the same organic ligand and metal ions as in Yang's report [50]. In a conventional three-electrode test cell, a specific capacitance of $988 \mathrm{~F} \mathrm{~g}^{-1}$ was achieved for Ni-MOF in $3 \mathrm{~mol} \mathrm{~L}^{-1} \mathrm{KOH}$ at $1.4 \mathrm{~A} \mathrm{~g} \mathrm{~g}^{-1}$. Notably, the capacitance retention was $96.5 \%$ after 5,000 cycles, which is close to that of some carbon electrode materials. Furthermore, a flexible solid-state asymmetric supercapacitor (ASC) was assembled with Ni-MOF as the positive electrode, and an activated carbon as negative electrode. The capacitance of the ASC was $230 \mathrm{mF} \mathrm{cm}^{-2}$ at a current density of $1 \mathrm{~mA} \mathrm{~cm}^{-2}$ (Fig. 5a). After 5,000 cycles, the capacitance retention was $92.8 \%$ (Fig. 5b). These $\mathrm{Ni}-\mathrm{MOF}$ showed excellent specific capacitance and stability, which was higher than that of previous metal compounds [52]. Jiao et al. [53] also reported the electrochemical properties of obtained Ni-MOF with a similar method. The specific capacitance of the Ni-MOF at a current density of $0.5 \mathrm{~A} \mathrm{~g}^{-1}$ was found to be $948 \mathrm{~F} \mathrm{~g}^{-1}$
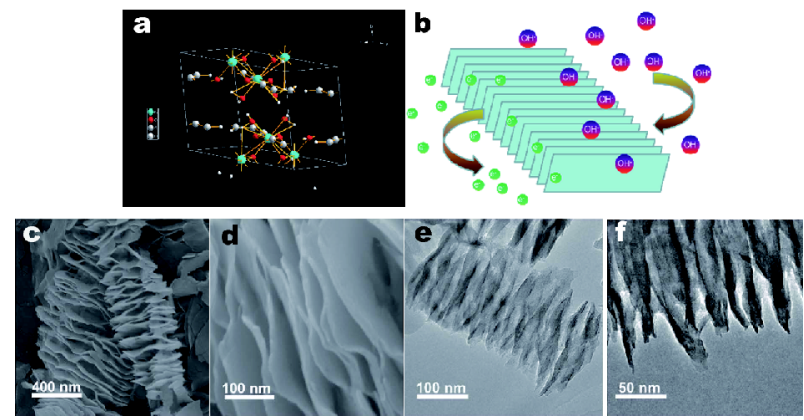

Figure 4 (a) The crystal structure of the Ni-MOF; (b) schematic representation of the proposed charge/discharge process; (c, d) SEM images of Ni-MOF; (e, f) transmission electron microscopy (TEM) images of Ni-MOF. Reproduced with permission from ref. [51], Copyright 2016, the Royal Society of Chemistry.
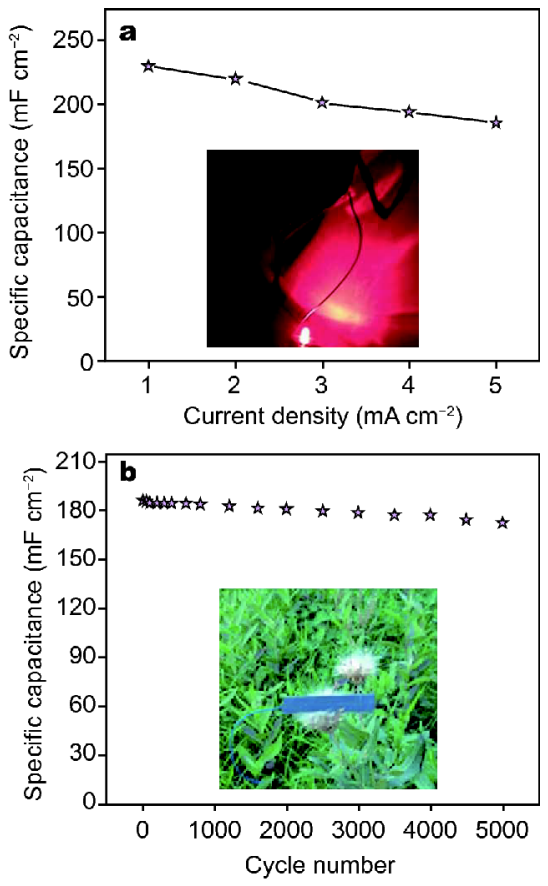

Figure 5 (a) The specific capacitance of the supercapacitor device at different current densities. The inset image is a photograph of the device. (b) Cycling performance of the device at $5.0 \mathrm{~mA} \mathrm{~cm}^{-2}$ after 5,000 cycles. The inset image is a photograph of the flexible ASC based Ni-MOF. Reproduced with permission from Ref. [51], Copyright 2016, the Royal Society of Chemistry.

with $3 \mathrm{~mol} \mathrm{~L}^{-1} \mathrm{KOH}+0.1 \mathrm{~mol} \mathrm{~L}^{-1} \mathrm{KOH}$ in $\mathrm{K}_{4} \mathrm{Fe}(\mathrm{CN})_{6}$ as the electrolyte.

$\mathrm{Xu}$ et al. [54] synthesized a high performance rod-like Ni-MOF via hydrothermal method using nickel nitrate and the organic ligand, salicylic acid. The SSA of the obtained Ni-MOF reached $186.8 \mathrm{~m}^{2} \mathrm{~g}^{-1}$. The specific capacitance of Ni-MOF was calculated to be $1,698 \mathrm{~F} \mathrm{~g}^{-1}$ in $6 \mathrm{~mol} \mathrm{~L}^{-1} \mathrm{KOH}$ electrolyte at $1 \mathrm{~A} \mathrm{~g}^{-1}$ using a three-elec- 

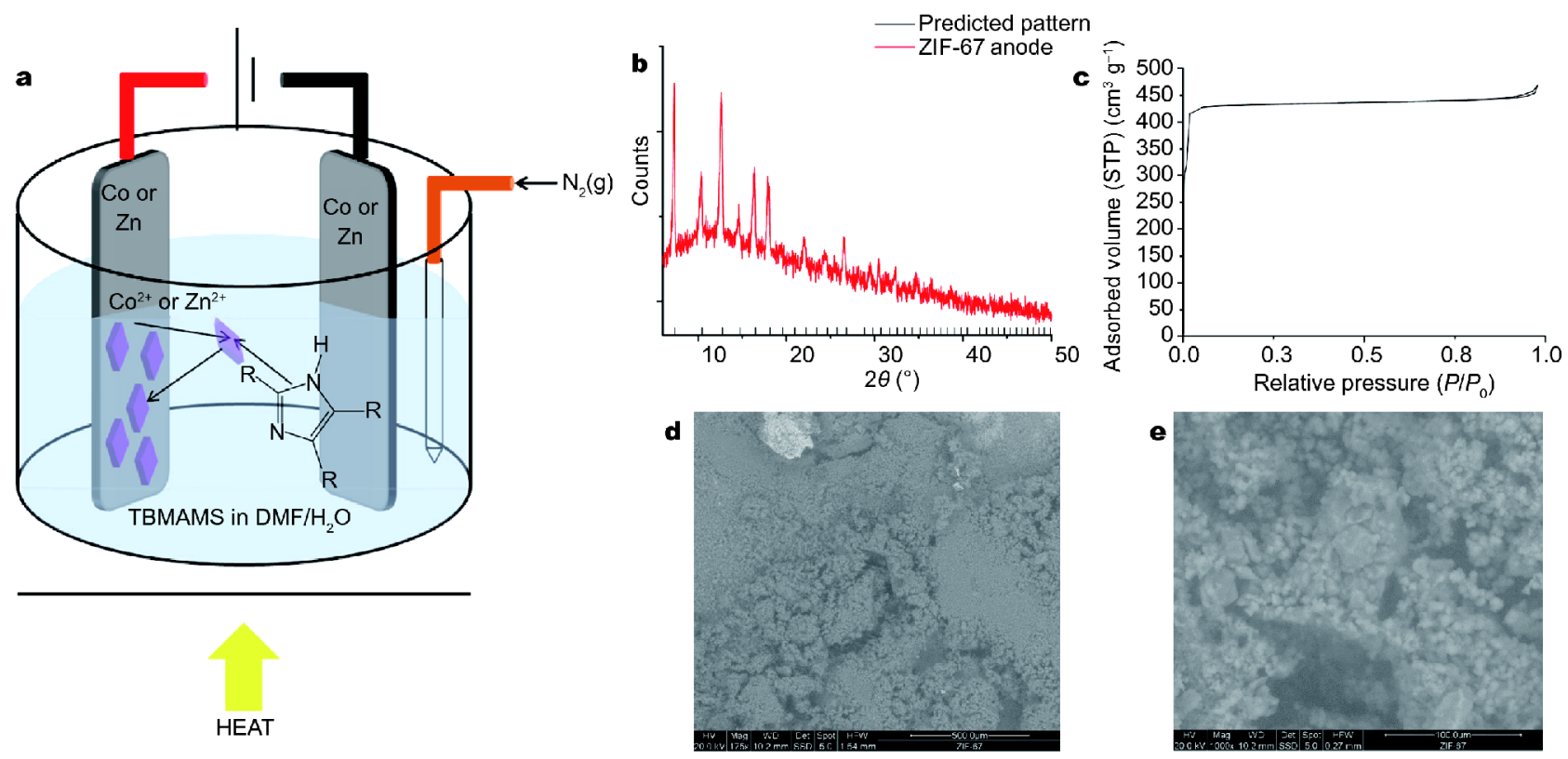

Figure 6 (a) An illustration of the experimental setup to fabricate the ZIF coatings; (b) powder XRD confirms the identity of the coating as ZIF-67 with the reflections in the experimental pattern (red) matching the predicted positions (black); (c) $\mathrm{N}_{2}$ adsorption and desorption curves of ZIF-67; (d) the coherency of the coating of ZIF-67 on the anode and (e) the lack of a clear morphology in the constituent crystals. Reproduced with permission from Ref. [55], Copyright 2016, Elsevier.

trode test cell. The excellent capacitance was ascribed to the high SSA, suitable pore size distribution and fast charge mobility of the rod-like Ni-MOF. In addition, the capacitance retention rate was $94.8 \%$ after 1000 cycles. Furthermore, the specific capacitance of an ASC fabricated from Ni-MOF and graphene electrodes was $166 \mathrm{~F} \mathrm{~g}^{-1}$ at $1 \mathrm{~A} \mathrm{~g}^{-1}$.

Currently, Ni-MOFs generate a lot of research interest given their promising electrochemical performance. As described above, they are applied as supercapacitor electrode materials and can display high specific capacitances and exceptional retention rates, justifying their position as a material of interest for this purpose.

Worrall et al. [55] prepared zeolitic imidazolate frameworks (ZIFs) with imidazole (IM), benzimidazole (bIM), methyl imidazole (mIM) and ethyl imidazole (eIM) as organic ligands via electrochemical coating (Fig. 6). Of the variations reported, the ZIF-67 electrodes displayed the highest areal capacitance of $10.45 \mathrm{mF} \mathrm{cm}^{-2}$ at a scanning rate of $10 \mathrm{mV} \mathrm{s}^{-1}$. In addition, the electrical conductivity of ZIF-67 was also observed to be the best out of the reported MOFs and graphene complexes, as can be seen from Table 1 .

Yang et al. [56] produced layered Co-MOFs using cobalt nitrate and PTA organic ligands (Fig. 7), and tested these in $5 \mathrm{~mol} \mathrm{~L}^{-1} \mathrm{KOH}$ electrolyte. A resulting capaci-
Table 1 Summary of the solution resistances $\left(R_{\mathrm{S}}\right)$ and charge transfer resistances $\left(R_{\mathrm{CT}}\right)$ calculated from the fitting of electrochemical impedance spectroscopy (EIS) data [55]

\begin{tabular}{ccc}
\hline & $R_{\mathrm{S}}(\Omega)$ & $R_{\mathrm{CT}}(\Omega)$ \\
\hline ZIF-zni & 2.10 & 62,600 \\
ZIF-7 & 2.25 & 86,100 \\
ZIF-8 & 3.45 & 3,740 \\
ZIF-14 & 3.21 & 8,320 \\
ZIF-67 & 2.77 & 21 \\
\hline
\end{tabular}

tance of $2,564 \mathrm{~F} \mathrm{~g}^{-1}$ was achieved at $1 \mathrm{Ag}^{-1}$, and a considerable $1,164 \mathrm{~F} \mathrm{~g}^{-1}$ was maintained as the current density was increased to $20 \mathrm{~A} \mathrm{~g} \mathrm{~g}^{-1}$. Compared with the NiMOFs reported above, this type of Co-MOF demonstrated relatively similar cyclic stability, achieving $95.8 \%$ after 3,000 cycles. The authors found that there was enough space for electrolyte storage within the large interlayer distance of Co-MOFs, which endowed them with more facile $\mathrm{OH}^{-}$intercalation and deintercalation, improving the capacitance of the MOFs (Fig. 7c). The prepared Co-MOFs also displayed excellent conductivity due to the conductive network, as shown in Fig. $7 \mathrm{~d}$. The conductive network structure (allowing fast and efficient electron transfer) as well as the diffusion enabling geometries (the layered structure and nanosheet morphology) are key aspects that enhanced the capacitive 

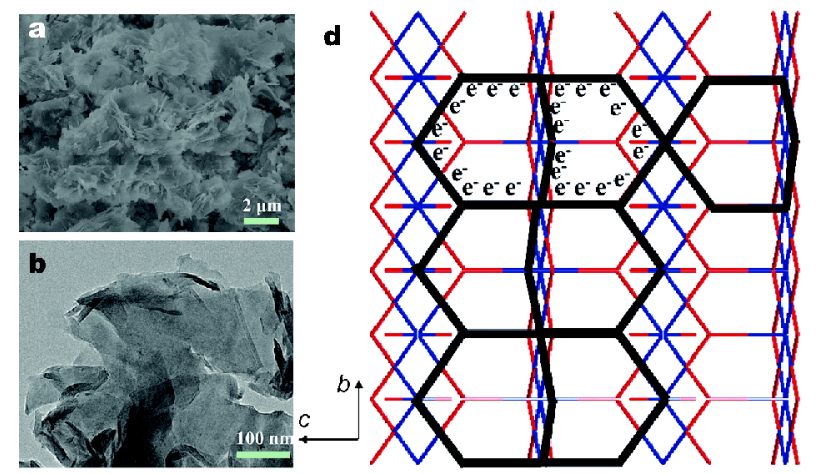

c

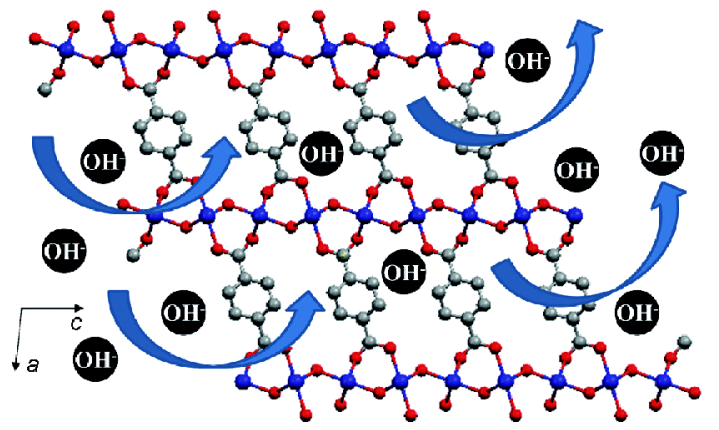

Figure 7 (a) SEM image of Co-MOFs; (b) TEM image of Co-MOFs; (c) the inter/deintercalation process of $\mathrm{OH}^{-}$in the layered structure of the Co-MOF crystal; (d) the electron transport process in the conductive network of the Co-MOF crystal. Reproduced with permission from Ref. [56], Copyright 2017, Wiley-VCH Verlag GmbH \& Co. KGaA.

performance of Co-MOFs in this study.

Sheberla et al. [57] constructed honeycomb-like structured Ni-MOFs (Fig. 8) using nickel and 2,3,6,7,10,11hexaiminotriphenylene $\left(\mathrm{H}_{6} \mathrm{HITP}\right)$. The Ni-MOFs showed excellent EDLC performance in an ionic liquid electrolyte. The specific capacitance of Ni-MOFs was measured to be $111 \mathrm{~F} \mathrm{~g}^{-1}$ in $1 \mathrm{~mol} \mathrm{~L}^{-1} \mathrm{TEABF}_{4} / \mathrm{CAN}$ electrolyte at $0.05 \mathrm{~A} \mathrm{~g}^{-1}$, together with a surface area normalized capacitance of $18 \mu \mathrm{F} \mathrm{cm}^{-2}$, which is greater than that of most carbon materials. The improved EDLC of Ni-MOFs is due to the appropriate pore diameter and good electrical conductivity. Evidently, the columnar voids and the electrolyte ions were consistent with the appropriate pore sizes $(1.5 \mathrm{~nm})$ of the Ni-MOFs [58]. In addition, the presence of conjugated $\pi$ bonds is also a key aspect that increases the charge mobility and further enhances the electrical conductivity of the as-prepared Ni-MOFs [31].

\section{MOF DERIVED ELECTRODE MATERIALS}

\section{Porous carbons}

MOFs have been widely investigated over the past two decades given their intrinsic ordered porous network structure, high SSA and uniform pore size distribution.
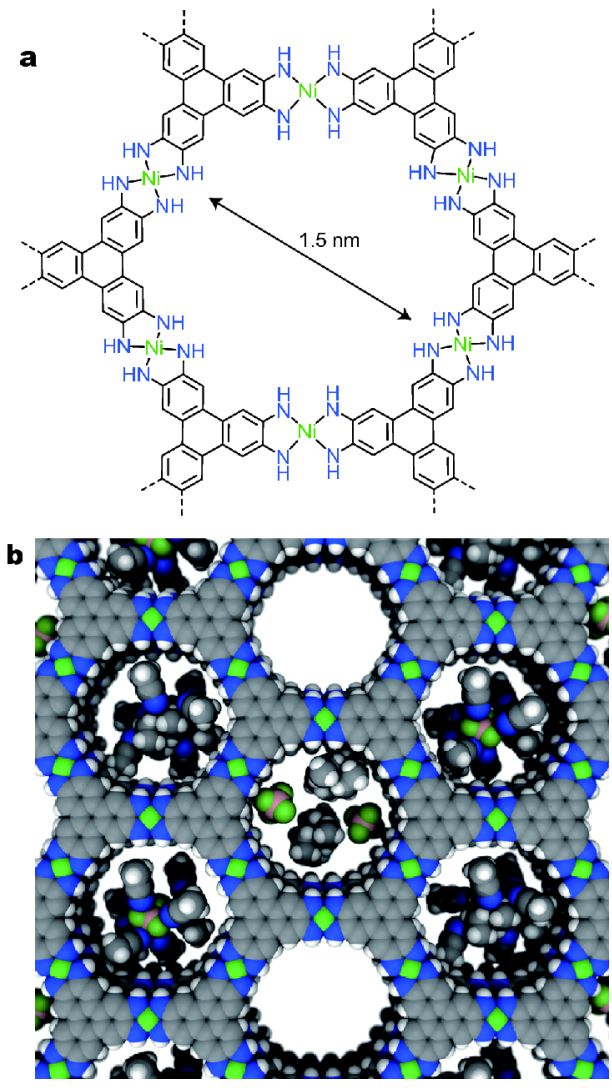

Figure 8 (a) Molecular structure of Ni-MOF; (b) relative size of pores, electrolyte $\mathrm{Et}_{4} \mathrm{~N}^{+}$and $\mathrm{BF}_{4}^{-}$, and acetonitrile solvent molecules shown in a space-filling diagram of idealized Ni-MOF. Ni: green; F: lime; N: blue; C: gray; B: brown; H: white. Reproduced with permission from Ref. [57], Copyright 2017, Nature Publishing Group.

However, there remain limitations in the application of MOFs. Specifically, MOFs possess relatively poor thermal and chemical stability, due to their skeleton structure. This drawback greatly limits the application of MOFs in industrial settings, and remains a key challenge to be addressed. To address the issue, many researchers have focused on the design and synthesis of highly stable MOFs. Alternatively, some researchers began to consider how the poor stability and easy decomposition characteristics of MOFs may be improved. As a result, versatile nanoporous carbon materials have been prepared through the carbonisation of MOFs, and these are able to retain high SSA and uniform pore size distribution. The high SSA and uniform pore size distribution are beneficial to the contact between the electrode and the electrolyte as well as accelerating the transfer of electrolyte ions, potentially enhancing the EDLC. There are two common methods for preparing porous carbon materials by MOFs. (1) The MOF backbones act as a carbon source, 

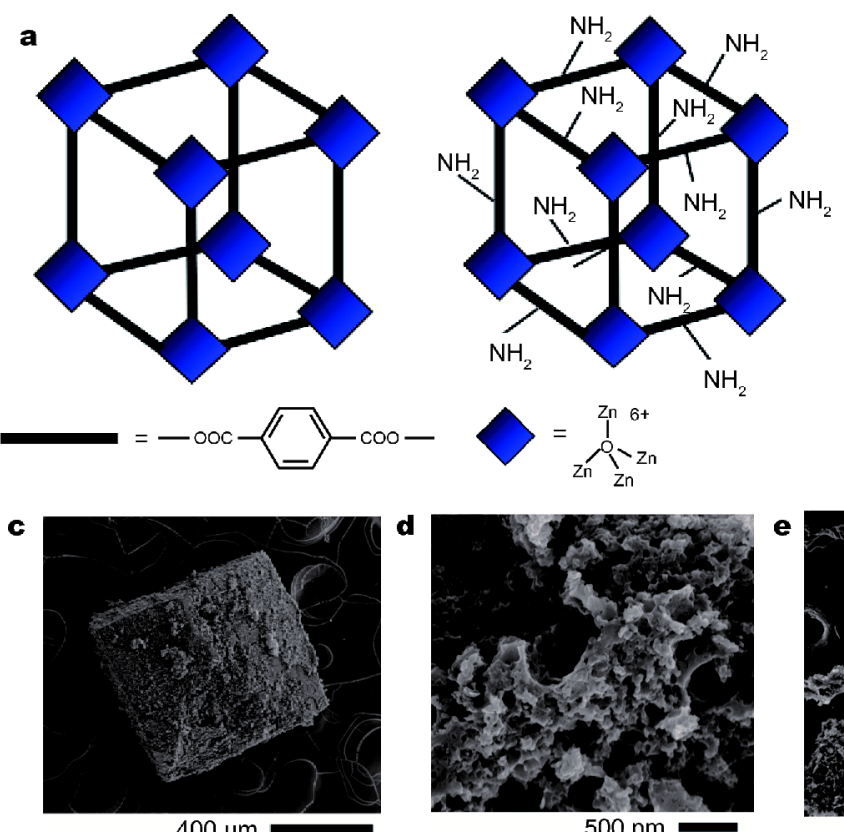

$500 \mathrm{~nm}$
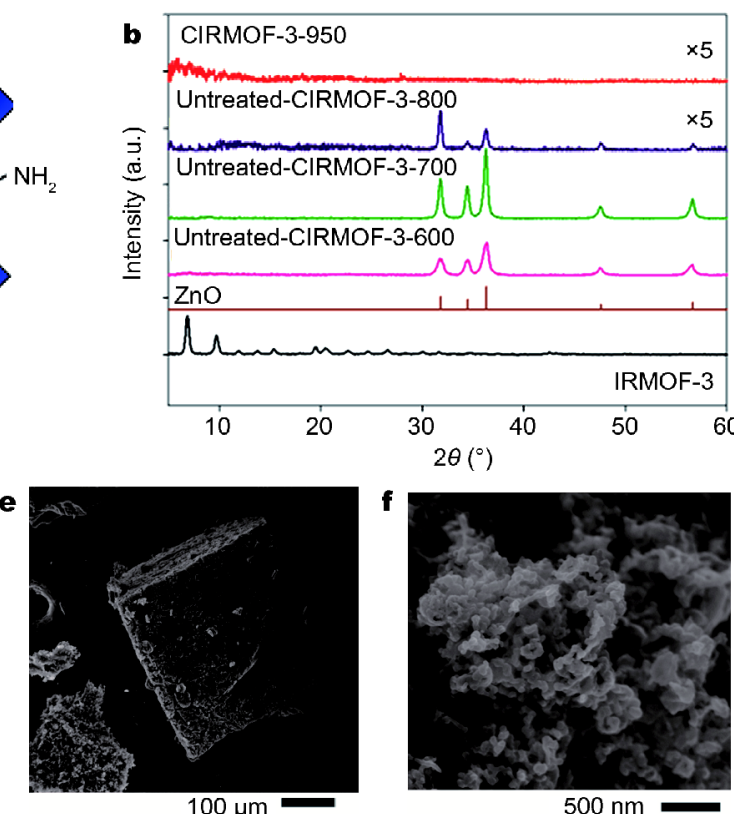

Figure 9 (a) Schematic structure of MOF-5 and IRMOF-3; (b) XRD patterns; (c, d) SEM images of CIRMOF-3-950; (e, f) SEM images of CMOF-5950. Reproduced with permission from Ref. [59], Copyright 2014, American Chemical Society.

and are directly carbonized to obtain carbon material. (2) Adding other carbon sources to MOFs, and then carbonizing them into carbon material.

\section{MOFs used as carbon sources}

Generally, porous carbon materials can be obtained by carbonization and acid treating of MOFs because the low boiling point of metal center, such as $\mathrm{Zn}$ (i.e., $907^{\circ} \mathrm{C}$ ) in Zn-MOF.

Nitrogen doped isoreticular MOFs (IRMOF-3) (Fig. 9a) were prepared via solvothermal approaches using zinc nitrate and $\mathrm{H}_{2} \mathrm{DBC}, 2$-aminobenzene-1,4,-dicarboxylate by Jeon et al. [59]. The material was subsequently converted to a nitrogenous porous carbon IRMOF, or carbonized IRMOF (CIRMOF), by thermal treatment. Compared to MOF-5 which was subjected to similar thermal carbonization treatment, denoted as CMOF, the SSA of the precursor MOF-5 and IRMOF-3 was found to be 1,305 and $1,221 \mathrm{~m}^{2} \mathrm{~g}^{-1}$, respectively. The effect of carbonization temperature was investigated for the MOFs (Fig. 9c-f). The ideal temperature was found to be $950^{\circ} \mathrm{C}$ considering capacitive performance. After carbonization, the SSA of carbonized MOFs at $950^{\circ} \mathrm{C}$ (CMOFs-950) and carbonized isoreticular MOFs at $950^{\circ} \mathrm{C}$ (CIRMOF-3-950) was 572 and $553 \mathrm{~m}^{2} \mathrm{~g}^{-1}$, respectively. It can be seen from the XRD pattern that the characteristic peaks of $\mathrm{ZnO}$ disappeared after $950^{\circ} \mathrm{C}$ annealing, proving that the
CMOFs-950 and CIRMOF-3-950 are carbon materials with no metal ions (Fig. 9b). A specific capacitance of $239 \mathrm{~F} \mathrm{~g}^{-1}$ was achieved for CIRMOF-3-950 in $1 \mathrm{~mol} \mathrm{~L}^{-1}$ $\mathrm{H}_{2} \mathrm{SO}_{4}$ electrolyte at $0.5 \mathrm{~A} \mathrm{~g}^{-1}$, which was greater than that of CMOFs-950 $\left(24 \mathrm{~F} \mathrm{~g}^{-1}\right)$. It is worth noting that the specific capacitance of CIRMOF-3-950 was not significantly reduced after 10,000 cycles, indicating the good cyclic and structural stability. Furthermore, when comparing the two carbon materials, the nitrogen-doped CIRMOF-3-950 material displayed higher capacitive performance, suggesting that the $\mathrm{N}$-doping has a positive effect.

Hao et al. [60] prepared nitrogen doped porous carbon materials through carbonizing and activating ZIF-11. Specifically, ammonium hydroxide was added to the reactant solution when synthesizing ZIF-11. The SSA of the prepared nitrogen doped porous carbon material was as high as $2,188 \mathrm{~m}^{2} \mathrm{~g}^{-1}$. This material also exhibited a high specific capacitance of $307 \mathrm{Fg}^{-1}$ in $1 \mathrm{~mol} \mathrm{~L}^{-1} \mathrm{H}_{2} \mathrm{SO}_{4}$ electrolyte at $1 \mathrm{~A} \mathrm{~g}^{-1}$.

Yang et al. [61] also obtained porous carbon materials by carbonizing Zn-MOF that was prepared through the reaction of zinc nitrate and different organic ligands, such as $\mathrm{H}_{2} \mathrm{BDC}, \mathrm{H}_{2} \mathrm{BPDC}$, trident trimesic acid $\left(\mathrm{H}_{3} \mathrm{BTC}\right)$ and 4,4',4"-benzene-1,3,5-triyl-tribenzoic $\left(\mathrm{H}_{3} \mathrm{BTB}\right)$ with the order of increasing length. It was found that the porous carbon prepared with $\mathrm{H}_{3} \mathrm{BDC}$ displayed the highest de- 
a

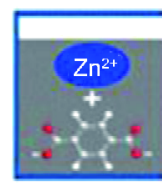

Terephthalic acid + Zinc nitrate
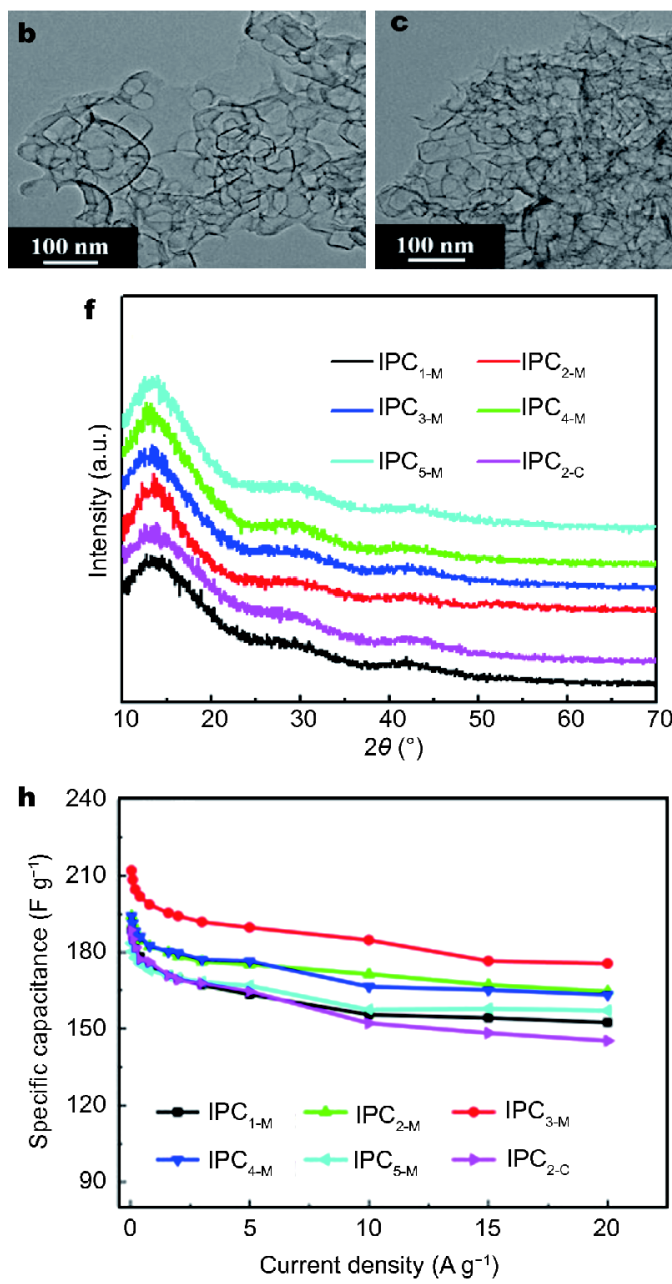

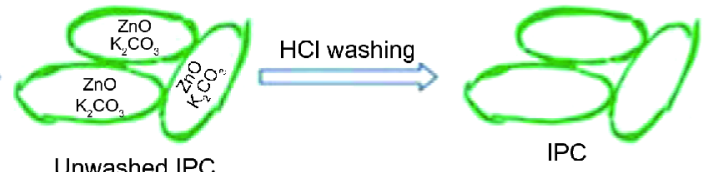

Unwashed IPC
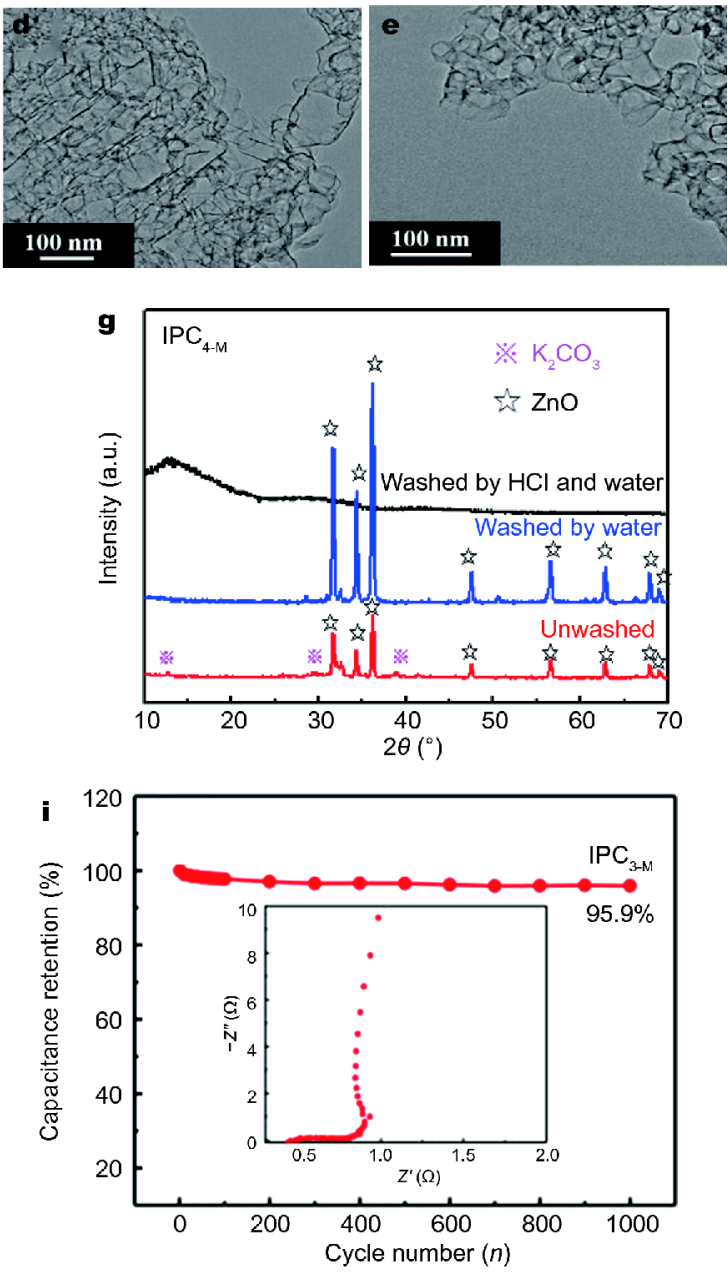

Figure 10 (a) Fabrication schematic of 3D IPCs. TEM images of $\operatorname{IPC}_{2-\mathrm{M}}$ (b), $\operatorname{IPC}_{3-\mathrm{M}}$ (c), $\operatorname{IPC}_{4-\mathrm{M}}$ (d) and IPC (d-M (e). (f) XRD patterns of IPCs; (g) XRD patterns of IPC $_{4-M}$ before and after washing; (h) specific capacitance of IPC electrodes at different current densities; (i) cyclic stability of IPC 3 -M. Reproduced with permission from Ref. [62], Copyright 2016, Elsevier.

gree of graphitization. In contrast to the results of Lee et al. [48], the porous carbon materials showed higher SSA as the length of the organic ligand chain, or "strut" length, decreased. Hence, the highest SSA of $2,816 \mathrm{~m}^{2} \mathrm{~g}^{-1}$ was measured for the porous carbon material using $\mathrm{H}_{2} \mathrm{BDC}$. A specific capacitance of $170 \mathrm{~F} \mathrm{~g}^{-1}$ was calculated for this material in $6 \mathrm{~mol} \mathrm{~L}^{-1} \mathrm{KOH}$ at a scan rate of $1 \mathrm{mV} \mathrm{s}^{-1}$, together with $98 \%$ capacitance retention after 5,000 cycles, indicating the stable and ordered structural porous carbon. The result shows that a large surface area, high porosity and high conductivity may enhance the capacitance of porous carbons.

$\mathrm{Yu}$ et al. [62] proposed a unique route to form 3D interconnected porous carbons (IPCs) derived from MOF-5 via microwave-assisted $\mathrm{KOH}$ activation (Fig. $10 \mathrm{a}-\mathrm{e})$. The MOF-5 was prepared by $\mathrm{H}_{2} \mathrm{BDC}$ and zinc nitrate at room temperature. Then, $\mathrm{KOH}$ was used to activate the MOF-5. Finally, acid etching was used to obtain IPCs. Fig. 10f shows the XRD patterns of IPCs with different MOF-5 to $\mathrm{KOH}$ mass ratios. It was found 
a
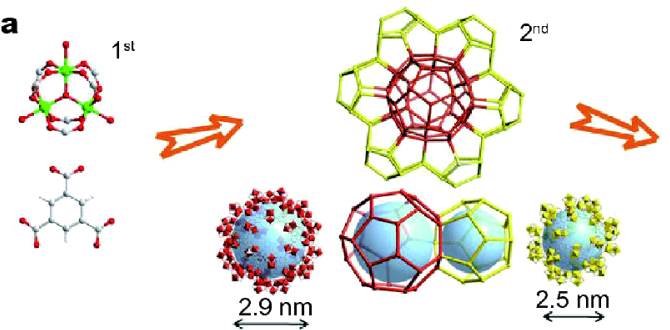

b

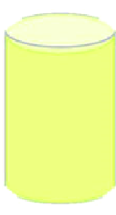

Wet gel
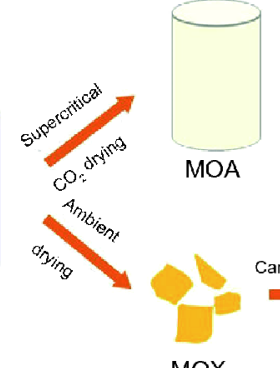

MOX

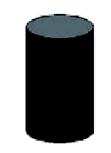

MOA-C-AI

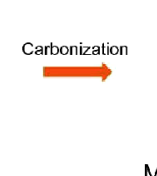

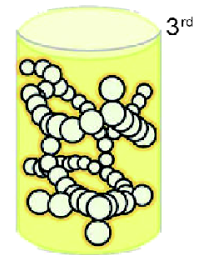

c

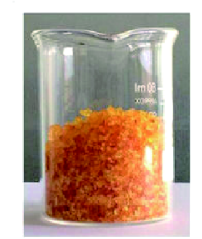

d

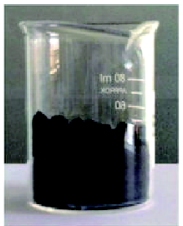

e

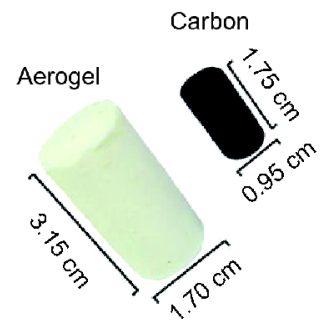

f

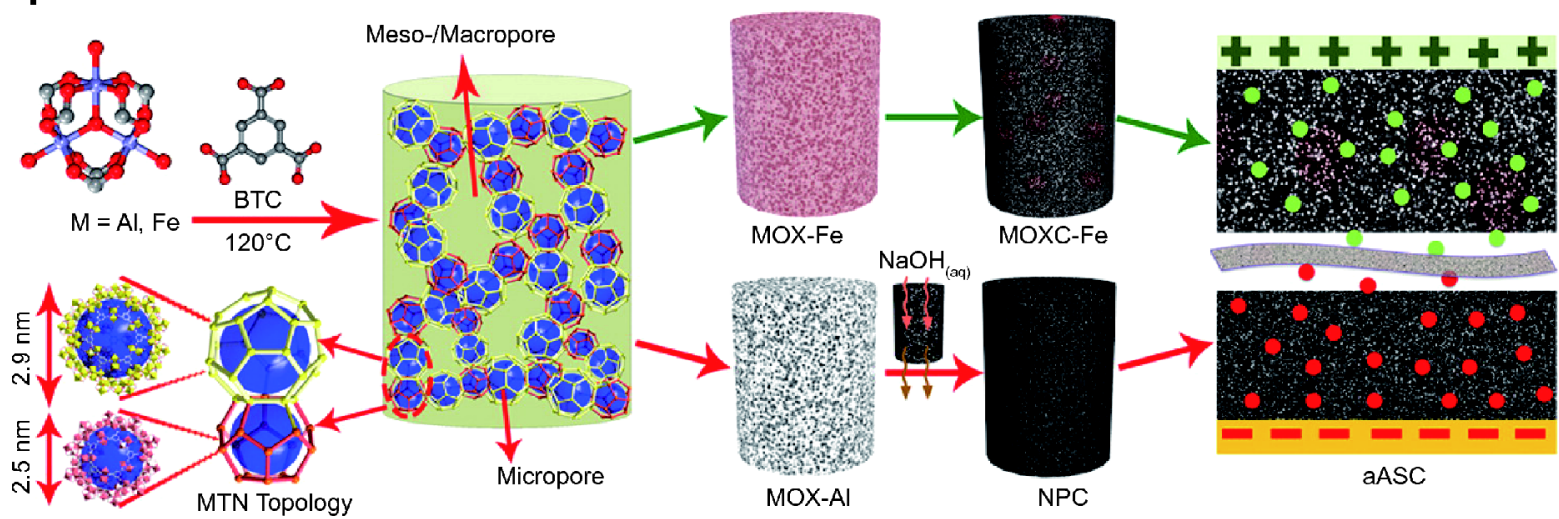

Figure $11(\mathrm{a}, \mathrm{b})$ Schematic of the fabrication of the Al-MOF, metal organic gels drying with supercritical $\mathrm{CO}_{2}(\mathrm{MOA})$ and after further carbonization (denoted as MOA-C), metal organic drying with air (MOX) and after further carbonization (denoted as MOX-C); (c, d) photographs of as-prepared MOX and final carbon products (MOX-C); (e) photographs of as-prepared MOA and MOA-C. Reproduced with permission from Ref. [63], Copyright 2013, Nature Publishing Group. (f) Schematic of the constructed ASC using nanoporous carbon and carbonized metal organic xerogels using $\mathrm{Fe}\left(\mathrm{NO}_{3}\right)_{3}(\mathrm{MOXC}-\mathrm{Fe})$, respectively, as electrode materials. Reproduced with permission from Ref. [64], Copyright 2016, American Chemical Society.

that the SSA of $\mathrm{IPC}_{4-\mathrm{M}}(\mathrm{MOF} / \mathrm{KOH}$ ratio was $4: 1)$ was the highest with $1,595 \mathrm{~m}^{2} \mathrm{~g}^{-1}$, and the associated pore distribution showed peaks around $0.4-0.9,1.1-1.8$ and $2-5 \mathrm{~nm}$. However, despite not having the greatest SSA, the specific capacitance of $\mathrm{IPC}_{3-\mathrm{M}}$ achieved the highest capacitance value of $212 \mathrm{~F} \mathrm{~g}^{-1}$ at $0.05 \mathrm{~A} \mathrm{~g}^{-1}$ in $6 \mathrm{~mol} \mathrm{~L}^{-1}$ $\mathrm{KOH}$ electrolyte (Fig. 10h). The capacitance retention rate for the material was $95.9 \%$ (Fig. 10i) after 1000 cycles. The relatively high electrochemical performance $\mathrm{IPC}_{3-\mathrm{M}}$ displayed was attributed to the bigger average pore size $(3.39 \mathrm{~nm})$, larger surface area $\left(1,515 \mathrm{~cm}^{2} \mathrm{~g}^{-1}\right)$ and lower internal resistance, charge-discharge resistance (Fig. 10i), which facilitated the ion diffusion as well as promoted the contact between electrolyte and electrode.

Xia et al. [63] obtained a whole piece of carbon aerogel material from a metal organic wet gel (Al-MOF), which was prepared using aluminum nitrate and $\mathrm{H}_{3} \mathrm{BTC}$ via a solvothermal approach. The Al-MOF was then dried in supercritical $\mathrm{CO}_{2}$, resulting in MOA. After further carbonization and hydrochloric acid etching to remove the $\mathrm{Al}$, a carbonaceous metal organic aerogel was formed which was denoted as MOA-C. For comparison in this study, MOX was formed by directly drying the Al-MOF in an oven at $80^{\circ} \mathrm{C}$. The material obtained after carbonization and activation, was labelled as MOX-C (Fig. 11a-e). Meanwhile, Mahmood et al. [64] prepared carbon 

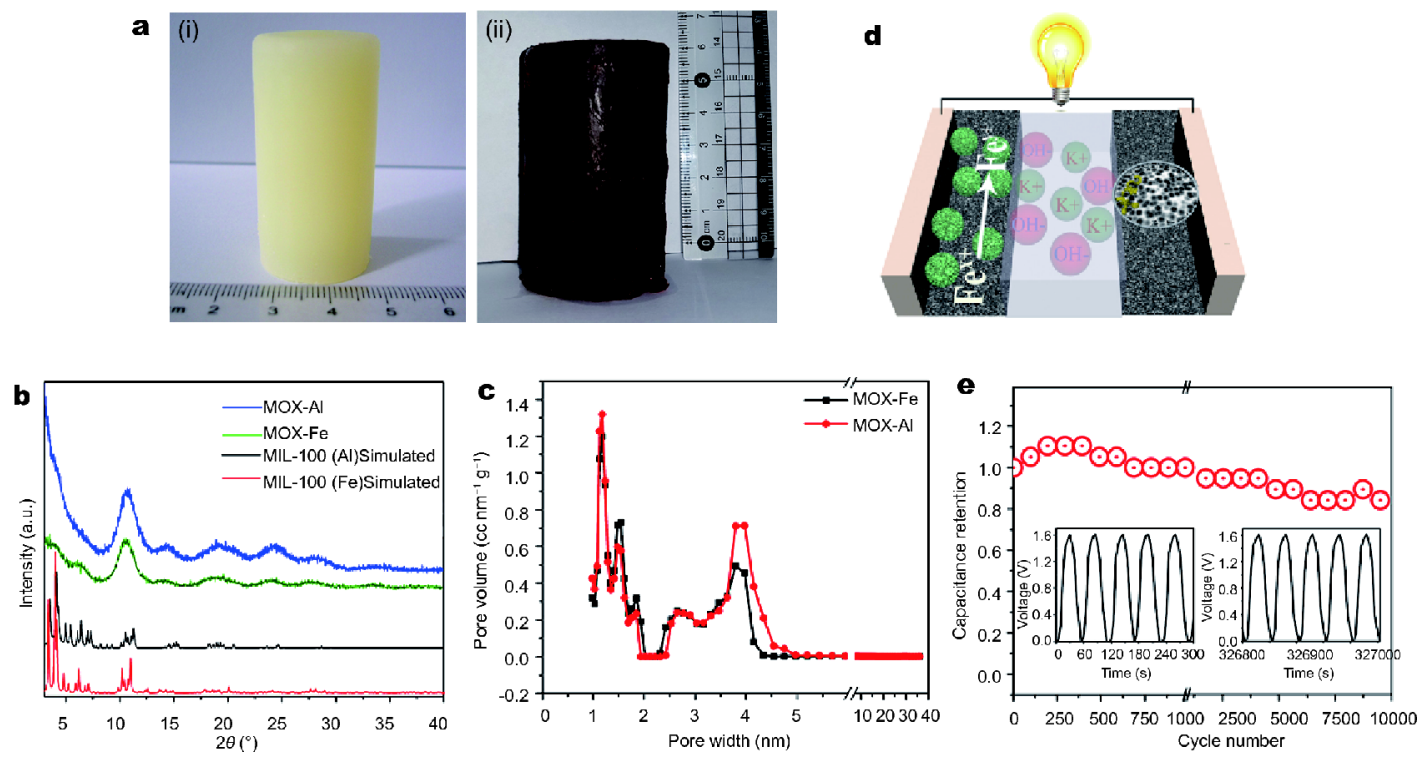

Figure 12 (a) Photographs of MOX-Al (i) and MOX-Fe (ii); (b) XRD patterns of MOX-Al and MOX-Fe; (c) pore size distribution of MOXs; (d) the schematic diagram of ASC; (e) the cyclic stability of ASC. Reproduced with permission from Ref. [64], Copyright 2016, American Chemical Society.

gel using a similar method, schematically represented in Fig. 11f. The electrochemical properties of MOXC-Fe and nanoporous carbon were investigated not only in a three electrode cell but also an ASC constructed from these two materials. MOXC-Fe exhibited specific capacitance of $600 \mathrm{~F} \mathrm{~g}^{-1}$ in $6 \mathrm{~mol} \mathrm{~L}^{-1} \mathrm{KOH}$ electrolyte, at a current density of $1 \mathrm{~A} \mathrm{~g}^{-1}$. The high performance is attributed to the improved pseudocapacitance derived from $\mathrm{Fe} / \mathrm{Fe}$ oxide transitions, in combination with EDLC provided by the carbon material framework. The stability of MOXCFe carbonized at $700^{\circ} \mathrm{C}$ was enhanced due to a near ideal thickness of carbon material decorating the $\mathrm{Fe}_{3} \mathrm{O}_{4} / \mathrm{Fe}$ metallic centers of the framework. Furthermore, the nanoporous carbon exhibited a specific capacitance of $207 \mathrm{~F} \mathrm{~g}^{-1}$ in $1 \mathrm{~mol} \mathrm{~L}^{-1} \mathrm{KOH}$ electrolyte at a scanning rate of $250 \mathrm{mV} \mathrm{s}^{-1}$. The EDLC was improved due to the higher surface area and hierarchical pore size distribution. The high SSA of $1,757 \mathrm{~m}^{2} \mathrm{~g}^{-1}$ for nanoporous carbon was ascribed to $\mathrm{NaOH}$ activation. The electrochemical performance of nanoporous carbon in this article outperformed most of the carbon materials delivered from MOFs that have been previously reported $[5,65]$. The ASC not only displayed a relatively high specific capacitance of $202.5 \mathrm{~F} \mathrm{~g}^{-1}$ in $6 \mathrm{~mol} \mathrm{~L}^{-1} \mathrm{KOH}$ electrolyte at $0.5 \mathrm{~A} \mathrm{~g}^{-1}$, but also good cyclic stability with greater than $80 \%$ capacitance retention after 10,000 cycles (Fig. 12e). The excellent electrochemical performance of supercapacitors was ascribed to the higher capacitance performance of positive and negative electrode material. When combined together into an ASC device, the independent stability of each electrode will influence the overall stability of the supercapacitor device.

MOFs in combination with other materials as carbon sources

Although the organic ligands can serve as carbon precursor of MOFs, other carbon sources are also used to prepare porous carbon materials, resulting in unique porous and stable carbon materials.

Liu et al. [66] added furan methanol (FA) as a carbon source to the reaction solution of MOF- 5 and investigated the electrochemical properties of the prepared carbon materials, at different temperatures. Jin et al. [67] applied glucose as a carbon source with MOF-5 and then investigated its electrochemical performance with ionic liquid electrolyte. Lei et al. [68] combined the approach of mesoporous molecular sieves with ZIF-67. In this work a ZIF-67 coating with $\mathrm{SiO}_{2} / \mathrm{RF}$, which formed using tetraethylorthosilicate (TEOS), resorcinol and formaldehyde, was prepared through one-step method (denoted as ZSR). For comparison, ZIF-67/SiO $/ 2 / \mathrm{RF}-\mathrm{M}$ was prepared by two steps in which ZIF-67 was firstly formed and by further carbonization and removal of silica, an $\mathrm{N}$-doped carbon was obtained, which is denoted as carbon-ZSR. The material possesses an ordered mesoporous structure and large SSA, as can be seen in Fig. 13. The SSA of the carbon-ZSR was measured to be $683 \mathrm{~m}^{2} \mathrm{~g}^{-1}$ and the associated pore volume was $0.897 \mathrm{~cm}^{3} \mathrm{~g}^{-1}$. The pore size 
a
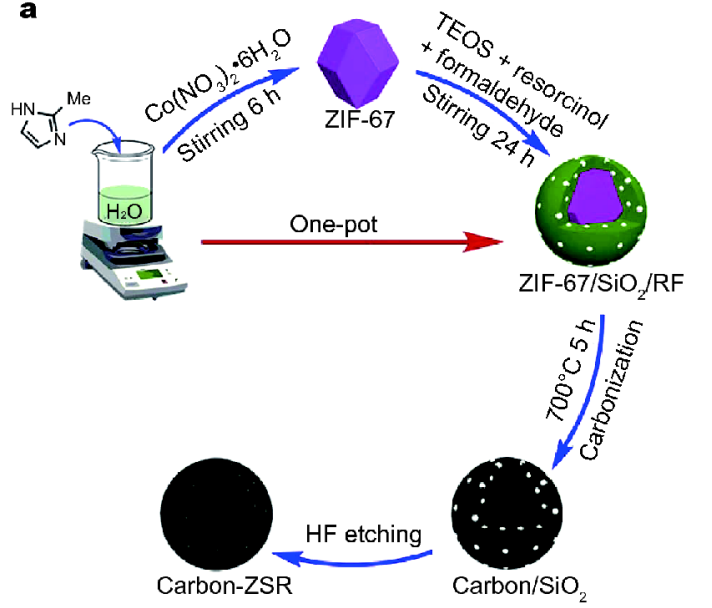

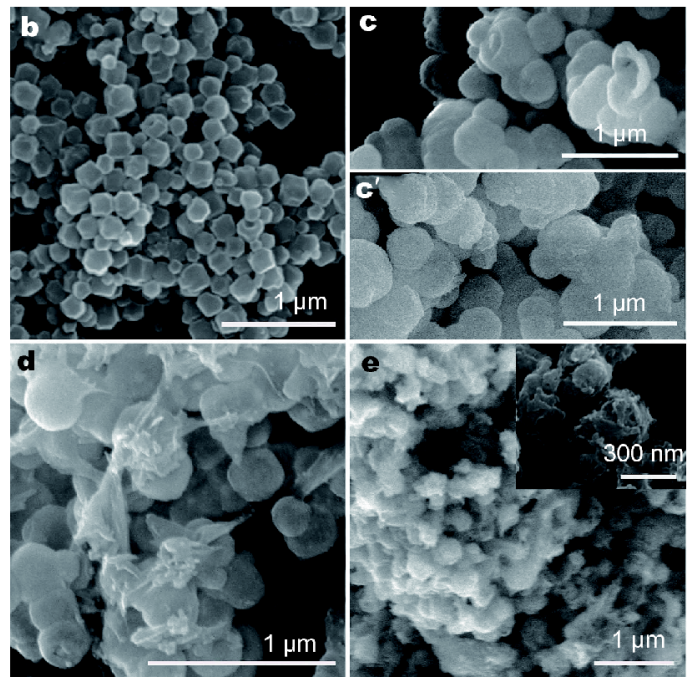

Figure 13 (a) Schematic illustration of the synthesis process of Carbon-ZSR; (b) SEM image of ZIF-67; (c) SEM images of ZIF-67/SiO $/ 2$ RF and (c') $\mathrm{ZIF}-67 / \mathrm{SiO}_{2} / \mathrm{RF}-\mathrm{M}$; (d) SEM image of carbon/SiO${ }_{2}$; (e) SEM images of carbon-ZSR. Reproduced with permission from Ref. [68], Copyright 2017, Elsevier.
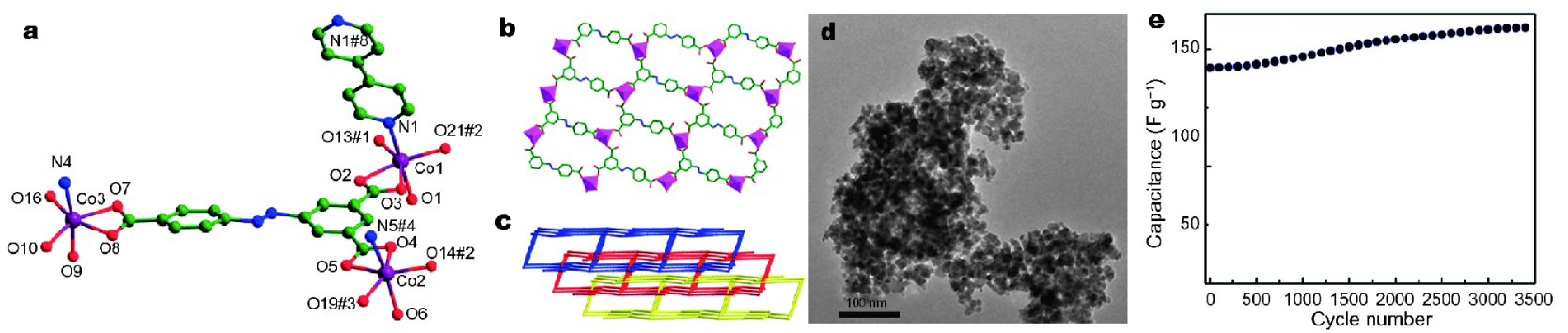

Figure 14 The coordination environment of the $\mathrm{Co}^{\mathrm{II}}$ ion (a). (b, c) 2D layer structure and 3D polycatenation array supramolecular architecture of Co-MOF; (d) TEM image of $\mathrm{Co}_{3} \mathrm{O}_{4}$; (e) cyclic stability of porous $\mathrm{Co}_{3} \mathrm{O}_{4}$ at $1 \mathrm{~A} \mathrm{~g}^{-1}$. Reproduced with permission from Ref. [76], Copyright 2013, the Royal Society of Chemistry.

distribution showed a strong peak at $3.96 \mathrm{~nm}$. Electrochemical performance testing resulted in a specific capacitance of $305 \mathrm{~F} \mathrm{~g}^{-1}$ in $1 \mathrm{~mol} \mathrm{~L}^{-1} \mathrm{H}_{2} \mathrm{SO}_{4}$ electrolyte at $1 \mathrm{~A} \mathrm{~g}^{-1}$, together with a retention rate of $98.4 \%$ after 5,000 cycles at $10 \mathrm{~A} \mathrm{~g}^{-1}$.

\section{Metal compounds (metal oxides or metal sulphides)}

Transition metal oxides are promising candidate for supercapacitor electrodes due to the multiple oxidation states/structures that enable rich redox reactions and high specific capacitance [69-71]. Thus, enormous effort has been made to design a robust and stable structure which lead to the superior energy density and long lifetime of metal compounds $[72,73]$. The metal compound prepared by annealing MOFs shows a relatively high cyclic stability than the other methods due to the pore shape and unique structure $[74,75]$.

Meng et al. [76] obtained $\mathrm{Co}_{3} \mathrm{O}_{4}$ from Co-MOF via a solvothermal method, followed by low temperature annealing. The unique Co-MOF crystal was formed by cobalt nitrate, azobenzene-3,5,4'-tricarboxylic acid $\left(\mathrm{H}_{3} \mathrm{ATBC}\right.$ ), and 4,4'-bipyridine (BPY) (Fig. 14a). The carboxylate groups of $\mathrm{H}_{3} \mathrm{ATBC}$ ligand were combined with $\mathrm{Co}$ (II) ions, resulting in the formation of a $2 \mathrm{D}$ layered structure (Fig. 14b). Furthermore, BPY ligand was used to combine the two layers with linear $\mathrm{N}, \mathrm{N}$-donor spacers to form an overall bi-layer gallery. In addition, the $2 \mathrm{D}$ bi-layer nets are then further stabilized by both $\pi-\pi$ stacking and hydrogen bonding interactions. Thus, the overall 3D polycatenation (Fig. 14c) array superstructure was formed. The SSA of $\mathrm{Co}_{3} \mathrm{O}_{4}$ was $47.12 \mathrm{~m}^{2} \mathrm{~g}^{-1}$ and pore diameter was measured to be $10.8 \mathrm{~nm}$. The specific capacitance reached $150 \mathrm{~F} \mathrm{~g}^{-1}$ in $2 \mathrm{~mol} \mathrm{~L}^{-1} \mathrm{KOH}$ electrolyte at $1 \mathrm{~A} \mathrm{~g}^{-1}$. Remarkably, there was no significant capacitance loss observed over 3,400 cycles at $1 \mathrm{~A} \mathrm{~g}^{-1}$ (Fig. 14d, e), thereby demonstrating excellent cyclic stability, which 

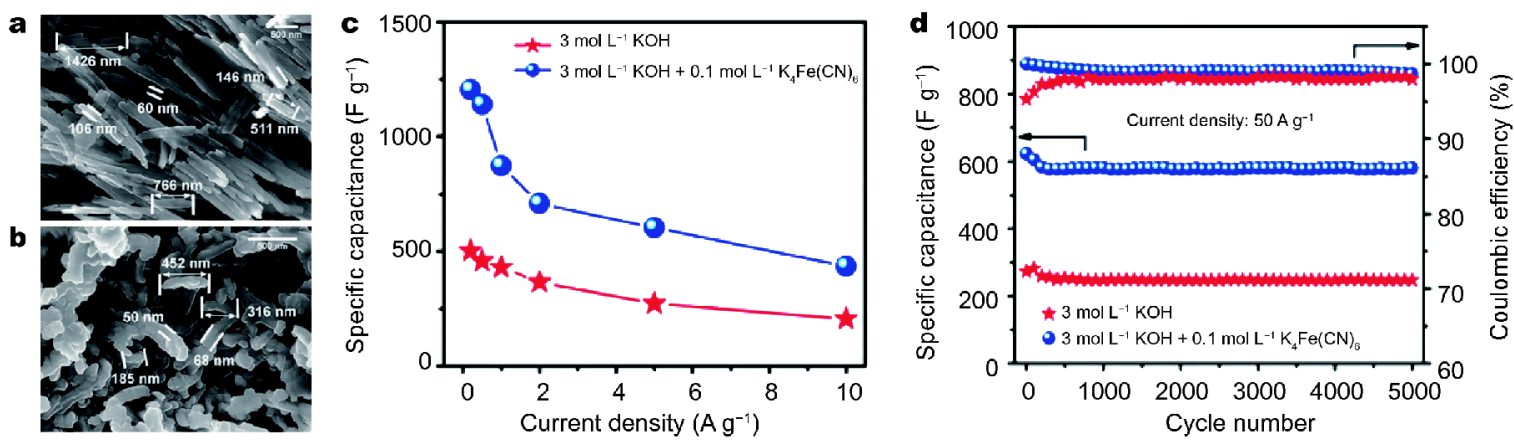

Figure 15 SEM images of Ce-MOF (a) and $\mathrm{CeO}_{2}$ (b). (c) Specific capacitance of $\mathrm{CeO}_{2}$ in different electrolytes. (d) Cyclic stability of CeO in different electrolytes and coulombic efficiency. Reproduced with permission from Ref. [77], Copyright 2014, the Royal Society of Chemistry.

in turn suggests strong chemical structural stability. The diffusion kinetics within the electrode was enhanced by the porous spaces created by $\mathrm{Co}_{3} \mathrm{O}_{4}$ aggregates, which served as reservoirs for electrolyte ions. The amorphous porous structure of the $\mathrm{Co}_{3} \mathrm{O}_{4}$ materials also provides abundant electroactive surface sites for redox pseudocapacitance.

Maiti et al. [77] prepared brick-like $\mathrm{CeO}_{2}$ (Fig. 15a, b) by thermal treatment of Ce-MOF used as a sacrificial material at $650^{\circ} \mathrm{C}$. The well-defined, aligned nanobars of Ce-MOF (length: $0.5-1.0 \mu \mathrm{m}$, width: $50-100 \mathrm{~nm}$ ) were formed via solvothermal method with metal cerium salt and $\mathrm{H}_{3} \mathrm{BTC}$ [78]. The SSA of $\mathrm{CeO}_{2}$ was calculated to be $77 \mathrm{~m}^{2} \mathrm{~g}^{-1}$, while that for Ce-MOFs is $27.3 \mathrm{~m}^{2} \mathrm{~g}^{-1}$. The superior surface area of $\mathrm{CeO}_{2}$ is presumably owed to the unique brick-upon-tile morphology, which contained a large amount of voids. In $3 \mathrm{~mol} \mathrm{~L}^{-1} \mathrm{KOH}$ electrolyte, the specific capacitance of $\mathrm{CeO}_{2}$ was $502 \mathrm{~F} \mathrm{~g}^{-1}$ at $0.2 \mathrm{~A} \mathrm{~g}^{-1}$ (Fig. $15 \mathrm{c})$. The addition of $1 \mathrm{~mol} \mathrm{~L}^{-1} \mathrm{~K}_{4} \mathrm{Fe}(\mathrm{CN})_{6}$ into the $3 \mathrm{~mol} \mathrm{~L}^{-1} \mathrm{KOH}$ electrolyte resulted in a remarkably high capacitance of $1,204 \mathrm{~F} \mathrm{~g}^{-1}$ at $0.2 \mathrm{~A} \mathrm{~g}^{-1}$. To describe the mechanism for this increase, the $\mathrm{Fe}(\mathrm{CN})_{6}{ }_{6}^{4-} / \mathrm{Fe}(\mathrm{CN})_{6}{ }^{3-}$ redox-active couple in the electrolyte acts as an electron buffer source and further supplements the $\mathrm{Ce}^{\mathrm{IV}} / \mathrm{Ce}^{\mathrm{III}}$ redox reactions at the electrode/electrolyte interface. Furthermore, with the mixed electrolyte the capacitance showed no decrease after 5,000 cycles (Fig. 15d). The high rate capability and cyclic stability indicate that the addition of new redox couples can facilitate the ionic diffusion, thereby promoting the redox kinetics. In this work, the pseudocapacitance was improved due to the increased availability of electrons from the redox reaction.

Chen et al. [79] synthesized NiCo oxides by the annealing method, in the process of which $\mathrm{Co}$ and $\mathrm{Ni}$ act as the central metal ions (Fig. 16a). Different kinds of NiCo oxides were formed with different $\mathrm{Ni} / \mathrm{Co}$ ratios. A specific capacitance of $797 \mathrm{Fg}^{-1}$ (Fig. 16b) was achieved for $\mathrm{NiCo}_{2} \mathrm{O}_{4}(\mathrm{Ni} / \mathrm{Co}=1: 2)$ in $6 \mathrm{~mol} \mathrm{~L}^{-1} \mathrm{KOH}$ at $1 \mathrm{~A} \mathrm{~g}^{-1}$, which was ascribed to the abundant redox active sites of $\mathrm{NiCo}_{2} \mathrm{O}_{4}$. In addition, the specific capacitance remained over $500 \mathrm{~F} \mathrm{~g}^{-1}$ after 10,000 cycles, demonstrating a reasonably long cycle life (Fig. 16c).

Salunkhe et al. [80] prepared nanoporous carbon and nanoporous $\mathrm{Co}_{3} \mathrm{O}_{4}$ by annealing ZIF-67. The nanoporous carbon material was prepared by high temperature carbonization, while the nonporous $\mathrm{Co}_{3} \mathrm{O}_{4}$ by low temperature annealing in air (Fig. 17a). The nanoporous carbon and $\mathrm{Co}_{3} \mathrm{O}_{4}$ were used to assemble an ASC (Fig. 17c). The SSAs of nanoporous carbon and $\mathrm{Co}_{3} \mathrm{O}_{4}$ were approximately $350 \mathrm{~m}^{2} \mathrm{~g}^{-1}$ and $148 \mathrm{~m}^{2} \mathrm{~g}^{-1}$, respectively. In a threeelectrode configuration, the specific capacitance of $\mathrm{Co}_{3} \mathrm{O}_{4}$ reached $504 \mathrm{Fg}^{-1}$ in $6 \mathrm{~mol} \mathrm{~L}^{-1} \mathrm{KOH}$ electrolyte at $5 \mathrm{mV} \mathrm{s}^{-1}$ (Fig. 17b). The ASC displayed a specific capacitance of $101 \mathrm{~F} \mathrm{~g}^{-1}$ at $2 \mathrm{~A} \mathrm{~g}^{-1}$ in $6 \mathrm{~mol} \mathrm{~L}^{-1} \mathrm{KOH}$ electrolyte, together with $89 \%$ capacitance retention after 2,000 cycles.

Liu et al. [81] obtained $\mathrm{Co}_{9} \mathrm{~S}_{8} @ \mathrm{C}$ via carbonization of Co-MOF. Cobalt chloride, thiophene-2,5-dicarboxylate (TDC) and BPY were used to prepare Co-MOF. Following this, S-doped and $\mathrm{N}$-doped carbon cuboid $\left(\mathrm{Co}_{9} \mathrm{~S}_{8} @ \mathrm{SNCC}\right)$ and carbon bulk $\left(\mathrm{Co}_{9} \mathrm{~S}_{8} @ \mathrm{SNCB}\right)$ porous materials were formed by further hydrochloric acid etching (Fig. 18). $\mathrm{Co}_{9} \mathrm{~S}_{8} @ S N C B$ and $\mathrm{Co}_{9} \mathrm{~S}_{8} @ S N C C$ were prepared by hydrothermal method and solvothermal method, respectively. The SSA of $\mathrm{Co}_{9} \mathrm{~S}_{8} @ \mathrm{SNCC}$ and $\mathrm{Co}_{9} \mathrm{~S}_{8} @ \mathrm{SNCB}$ were measured to be 212.1 and $88.1 \mathrm{~m}^{2} \mathrm{~g}^{-1}$, respectively. The specific capacitance of $\mathrm{Co}_{9} \mathrm{~S}_{8} @ S N C C$ was $429 \mathrm{~F} \mathrm{~g}^{-1}$ which was higher than that of $\mathrm{Co}_{9} \mathrm{~S}_{8} @ \mathrm{SNCB}$ $\left(320 \mathrm{~F} \mathrm{~g}^{-1}\right)$. Both results were obtained in $6 \mathrm{~mol} \mathrm{~L}^{-1} \mathrm{KOH}$ electrolyte, at a current density of $1 \mathrm{~A} \mathrm{~g}^{-1}$. The relatively high capacitance of $\mathrm{Co}_{9} \mathrm{~S}_{8} @ S N C C$ is attributed to its higher SSA and unique porous structure which provides 

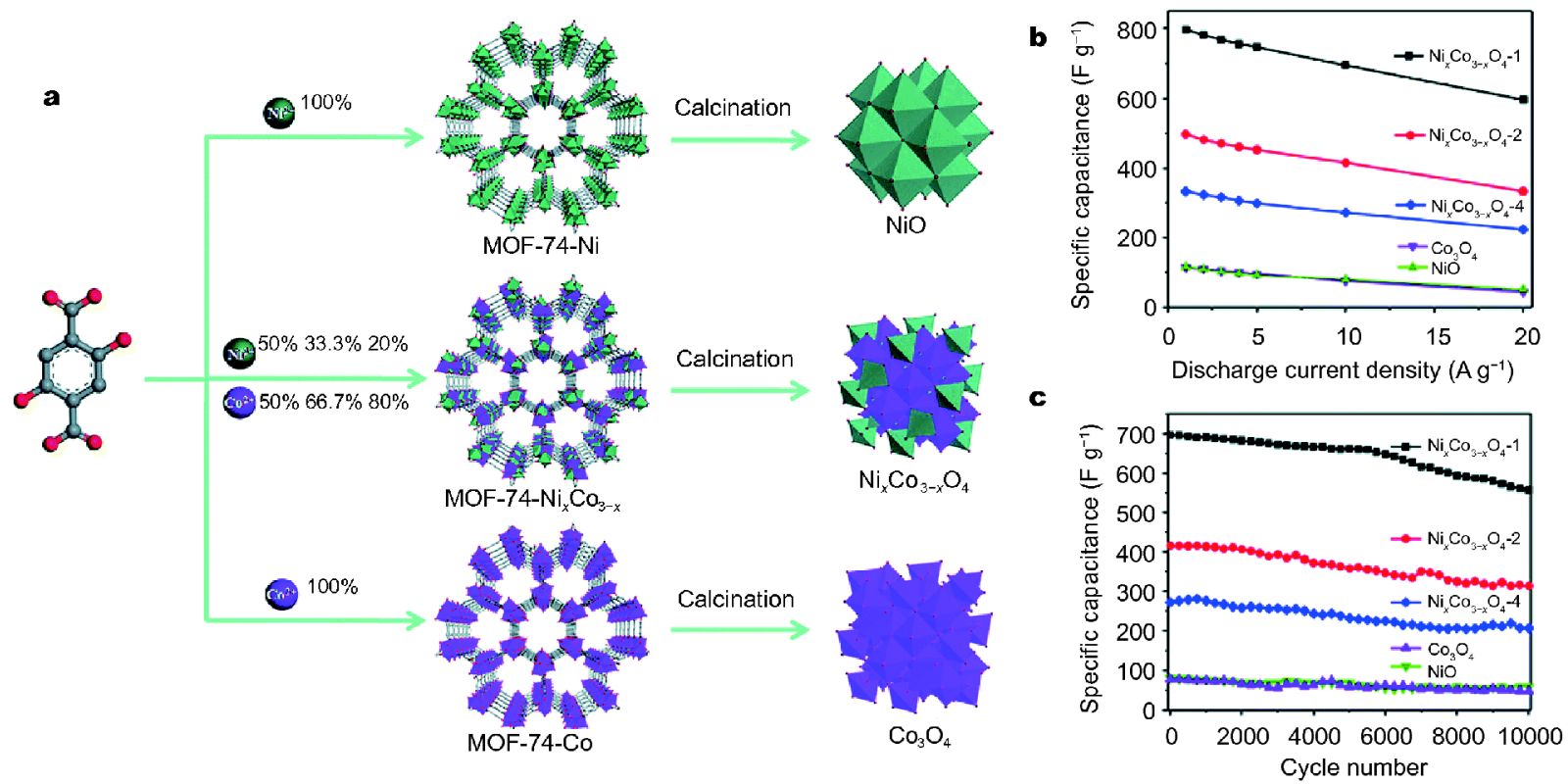

Figure 16 (a) Schematic of the fabrication process of NiCo oxide materials; (b) specific capacitance of different NiCo oxides at various current densities; (c) cyclic stability of NiCo oxides at the current density of $10 \mathrm{~A} \mathrm{~g}^{-1}$. Reproduced with permission from Ref. [79], Copyright 2015, the Royal Society of Chemistry.
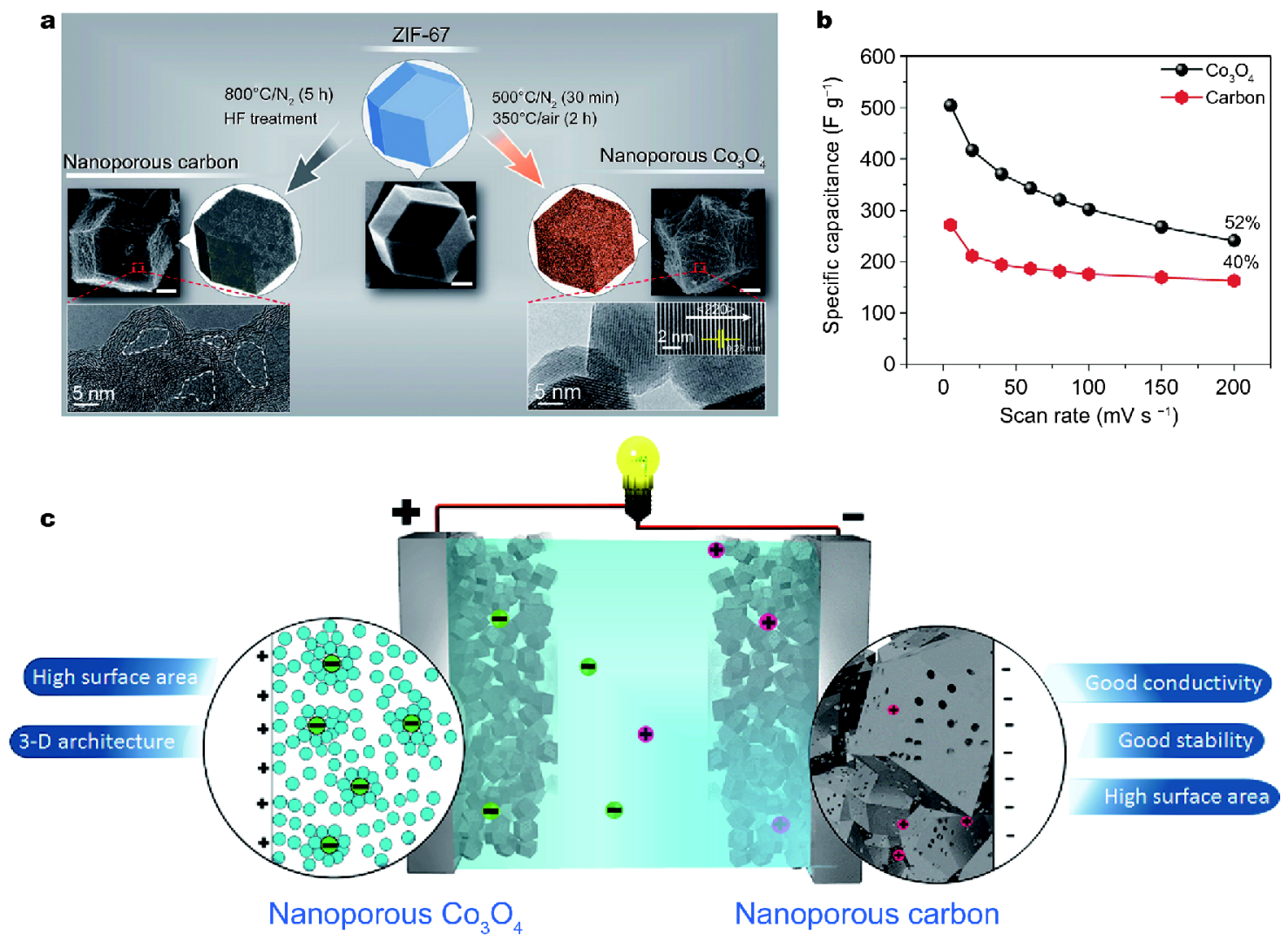

Figure 17 (a) The synthesis route of nanoporous carbon and $\mathrm{Co}_{3} \mathrm{O}_{4}$; (b) the specific capacitance of nanoporous carbon and $\mathrm{Co}_{3} \mathrm{O}_{4}$ at various scan rates; (c) schematic illustration of the fabricated ASC. Reproduced with permission from Ref. [80], Copyright 2015, American Chemical Society. 


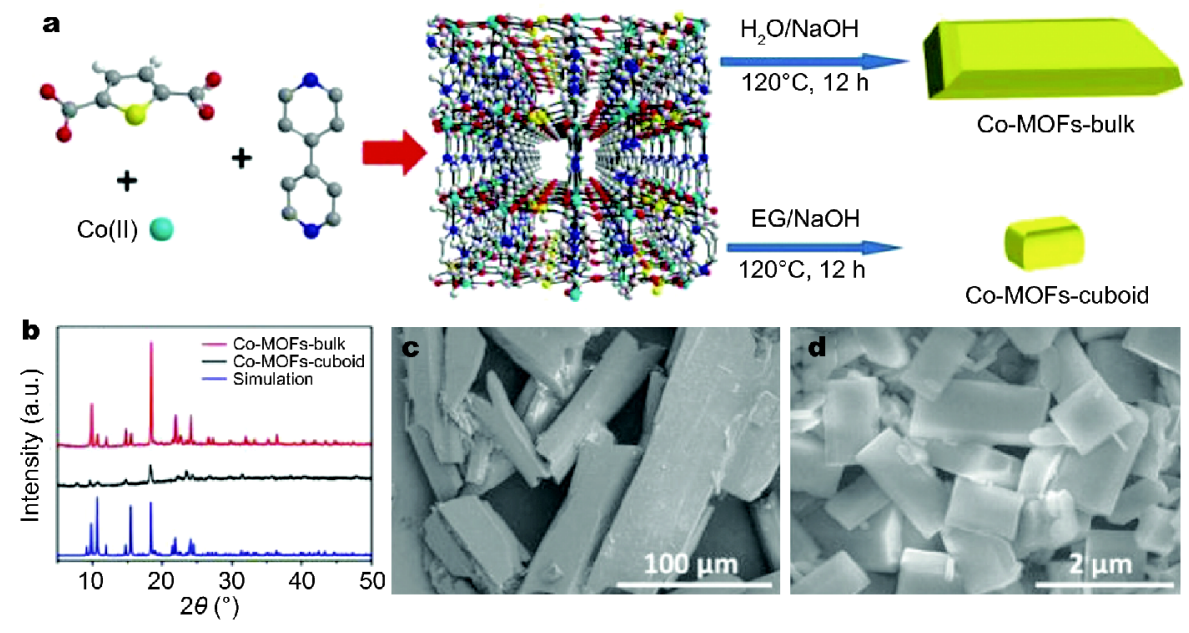

Figure 18 (a) Schematic illustration of Co-MOFs crystals. (b) XRD patterns. (c) SEM image of Co-MOF-bulk. (d) SEM image of Co-MOF-cuboid. Reproduced with permission from Ref. [81], Copyright 2017, the Royal Society of Chemistry.

sufficient space for electrolyte transport during charge/ discharge process. Furthermore, the intimate interaction between the graphitic carbon and $\mathrm{Co}_{9} \mathrm{~S}_{8}$ nanoparticles enables sufficient electron transport to the redox active sites [82-85]. After 5,000 cycles, the capacitance retention of $\mathrm{Co}_{9} \mathrm{~S}_{8} @$ SNCC was 98\%, demonstrating excellent cyclic stability.

\section{MOFs AND THEIR DERIVATIVES AS COMPOSITE SUPERCAPACITOR ELECTRODES}

Although MOFs display a large SSA and abundant active sites, the structure stability and electrical conductivity are not satisfactory. Adding surfactants and ion doping are effective strategies to construct a robust and stable structure of electrode materials. Furthermore, the low electrical conductivity increases irreversible capacitance and slows down the ion diffusion. Hence, conductive fillers like carbon materials and conductive polymers have been investigated to address these issues $[24,25,86]$. The composites can be prepared through a one-step method where the other materials participate in the reaction of preparing MOFs. On the other hand, the combination of MOFs and other materials can achieve after preparing the MOFs.

The composites of MOFs and additional materials used as supercapacitor electrodes

\section{Surfactant as additional material}

Surfactants play a key role in the synthesis of porous materials because they can act as sacrificial templates
$[87,88]$. In the process of synthesizing MOFs, surfactants can be used as a sacrificial template to obtain micropores and mesopores structure. Surfactants can be adsorbed onto the MOF structure acting as a structural guide agent during the synthesis of MOFs, creating an ordered pore structure [89]. There are three kinds of surfactants that are commonly used: polymer surfactants (triblock copolymer Pluronic F127, triblock copolymer P123, etc.), nonionic (polyols, amines), and ionic (alkyl quaternary ammonum salts) surfactants.

Meng et al. [90] synthesized spindle-like Fe-MIL-88B$\mathrm{NH}_{2}$ using the polymer surfactant $\mathrm{F} 127$ as a structuredirecting agent and template. The $\mathrm{Fe}_{3} \mathrm{O}_{4}$ /carbon composite was prepared by a well-controlled, incomplete annealing process (Fig. 19). The SSA of $37.7 \mathrm{~m}^{2} \mathrm{~g}^{-1}$ was achieved for the $\mathrm{Fe}_{3} \mathrm{O}_{4} /$ carbon composite. At $1 \mathrm{~A} \mathrm{~g}^{-1}$, the specific capacitances of this $\mathrm{Fe}_{3} \mathrm{O}_{4} /$ carbon were calculated to be $86,128,138,162 \mathrm{~F} \mathrm{~g}^{-1}$ in $1 \mathrm{~mol} \mathrm{~L}^{-1} \mathrm{KOH}$ electrolyte at $0,20^{\circ} \mathrm{C}, 40^{\circ} \mathrm{C}, 60^{\circ} \mathrm{C}$, respectively. During the first 1000 cycles at $20^{\circ} \mathrm{C}$, the specific capacitance of the electrode decreased slowly to $88 \%$ of the initial value, implying a somewhat stable electrode structure at $20^{\circ} \mathrm{C}$. However, it was observed that the capacitance increased rapidly when the temperature was raised to $40^{\circ} \mathrm{C}$, due to the decrease of internal resistance of electrolyte (Fig. 19e). After 1000 cycles at $40^{\circ} \mathrm{C}$, an ice-bath was used to reduce the temperature to $0^{\circ} \mathrm{C}$ and another 1000 charge/discharge cycles were performed. The temperature was then returned to $20^{\circ} \mathrm{C}$. After further 1000 cycles (4000 in total), the capacitance value of the electrode decreased to $83.3 \%$ of its initial capacity (Fig. 19f). The investigation of electrochemical performance at different temperatures lends 

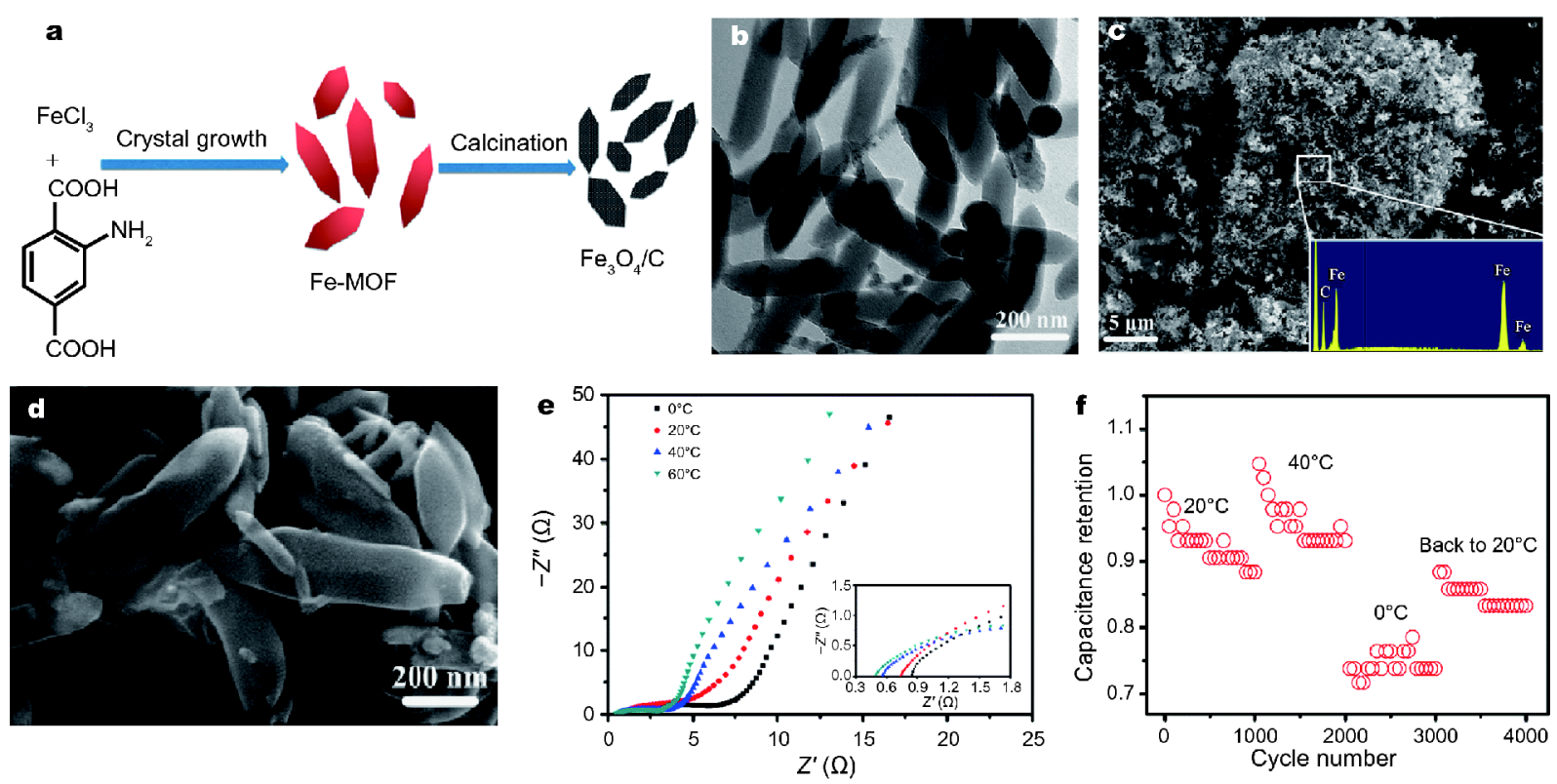

Figure 19 (a) Illustration of the fabrication of $\mathrm{Fe}_{3} \mathrm{O}_{4} /$ carbon composite; (b) TEM image of ion based MOF (Fe-MIL-88B-NH$)$; (c) SEM image and energy dispersive X-ray spectroscopy (EDS) spectrum of $\mathrm{Fe}_{3} \mathrm{O}_{4} /$ carbon composite; (d) SEM image of $\mathrm{Fe}_{3} \mathrm{O}_{4} /$ carbon composite; (e) EIS measurements of $\mathrm{Fe}_{3} \mathrm{O}_{4} /$ carbon composite electrode with different temperatures with insert showing the high frequency region of the Nyquist plot (enlarged profile showing as inset); (f) cyclic stability of $\mathrm{Fe}_{3} \mathrm{O}_{4} /$ carbon composite with varied temperature at $2 \mathrm{~A} \mathrm{~g}^{-1}$. Reproduced with permission from Ref. [90], Copyright 2014, Elsevier.

important insight into the suitability of an electrode material for practical applications. Therefore, it is of great significance to demonstrate that the prepared electrode materials were able to operate over a wide temperature range.

Salunkhe et al. [91] prepared nanoporous carbon materials by adding polyvinylpyrrolidone (PVP) to large particle ZIF-8 and then subjecting the mixture to further carbonization treatment. In this process, PVP acted as a structure-directing agent and participated in the formation of a ZIF-8 backbone. Cao et al. [92] also added PVP during the synthesis of porphyrin paddlewheel framework-3 (PPF-3) (Fig. 20). The PVP selectively attaches onto the surface of MOFs, controlling the vertical growth of PPF-3 MOF crystals and thereby resulting in the formation of 2D PPF-3 nanosheets. 2D CoSNC nanocomposites are then formed by a simultaneous sulfidation and carbonization process of the obtained 2D PPF-3 nanosheets (Fig. 20e). In the obtained CoSNC nanocomposites, the $\mathrm{CoS}_{1.097}$ nanoparticles were dispersed uniformly in the nitrogen-doped carbon matrix during the thermal treatment. This surfactant-assisted synthetic method is an efficient approach to construct $2 \mathrm{D}$ functional hybrid materials from MOF nanosheets with the specific capacitance found to be $360.1 \mathrm{~F} \mathrm{~g}^{-1}$ in $2 \mathrm{~mol} \mathrm{~L}^{-1}$ $\mathrm{KOH}$ electrolyte at $1.5 \mathrm{~A} \mathrm{~g}^{-1}$.
Metal materials as additional materials

Li et al. [93] reported novel hierarchical double-shelled $\mathrm{NiO} / \mathrm{ZnO}$ hollow spheres produced by a one-step solvothermal method, without any template, surfactant, or calcination (Fig. 21a). The Ni-Zn MOF was prepared with $\mathrm{H}_{2} \mathrm{BDC}$, zinc nitrate and nickel nitrate. The XRD pattern of Ni-Zn MOF matches well with the Zn-doped Ni-based MOF material [94], in which a double-layered structure with an enlarged interlayer distance was formed due to a chain like $\mathrm{M}(\mathrm{OH})_{3}$ secondary building unit of $\mathrm{Zn}$-doped $\mathrm{Ni}-\mathrm{MOF}$. The specific capacitance of $\mathrm{NiO} / \mathrm{ZnO}$ electrode was calculated to be $497 \mathrm{~F} \mathrm{~g}^{-1}$ in $3 \mathrm{~mol} \mathrm{~L}^{-1} \mathrm{KOH}$ electrolyte at $1.3 \mathrm{~A} \mathrm{~g}^{-1}$. Remarkably, the specific capacitance increased by $17.1 \%$ of its initial capacity after 2,000 cycles. The electrochemical performance benefited from the double shelled structure and high porosity, which facilitates the transfer of ions and electrons at the electrode/ electrolyte interface, and can also alleviate the volume change during the charge/discharge process.

Yang et al. [94] also fabricated layered structural Zndoped Ni-MOF by adding $\mathrm{Zn}$ ions into the reaction system of the MOF (Fig. 21b). The Zn-doped Ni-MOF structure becomes bigger as the doping amount of $\mathrm{Zn}$ increases, as the larger $\mathrm{Zn}^{2+}$ ions partially, or wholly replace the smaller $\mathrm{Ni}^{2+}$ ions. At $0.25 \mathrm{~A} \mathrm{~g}^{-1}$, the specific capacitance of MOF-2 ( $\mathrm{Zn}$-doped Ni-MOF with $\mathrm{ZnCl}_{2}$, 


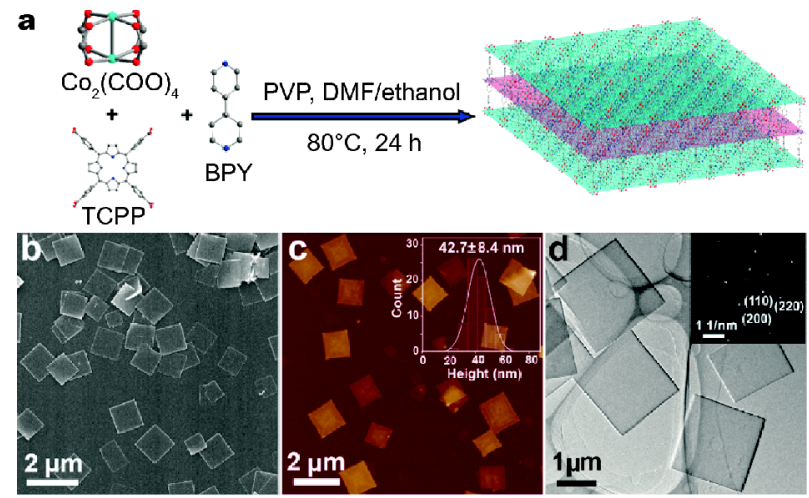

e

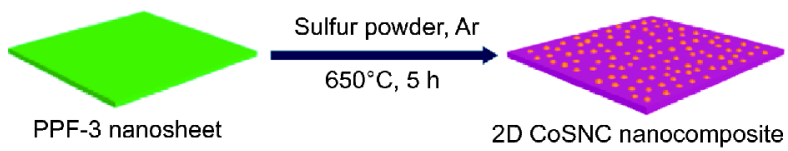

Figure 20 (a) Schematic illustrating the process of porphyrin paddlewheel framework-3 (PPF-3) nanosheets; (b) SEM image of PPF-3; (c) atomic force microscope (AFM) image of PPF-3; (d) TEM image of PPF-3, inset: selected area electron diffraction (SAED) pattern of PPF-3 nanosheets; (e) schematic for the synthesis process of 2D CoSNC nanocomposites. Reproduced with permission from Ref. [92], Copyright 2016, American Chemical Society.

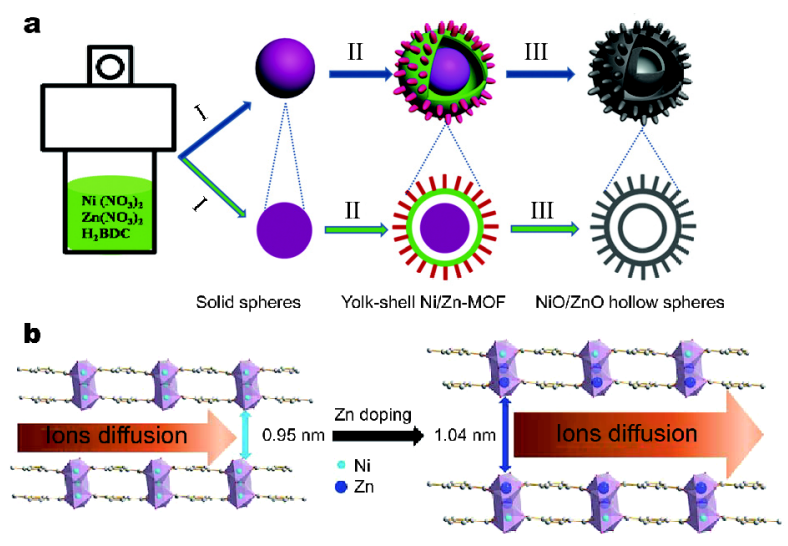

Figure 21 (a) Schematic of the synthesis of Ni/Zn-MOF microspheres and $\mathrm{NiO} / \mathrm{ZnO}$ hollow spheres. Reproduced with permission from Ref. [93], Copyright 2016, the Royal Society of Chemistry. (b) The structural change of the Ni-MOF before and after Zn-doping. Reproduced with permission from Ref. [94], Copyright 2014, the Royal Society of Chemistry.

$0.0456 \mathrm{~g}$ ) reached $1,620 \mathrm{Fg}^{-1}$ in $6 \mathrm{~mol} \mathrm{~L}^{-1} \mathrm{KOH}$ electrolyte. In addition, the specific capacity retention ratio was 91\% after 3,000 cycles. The results showed that MOF-2 not only displayed excellent capacitive performance, but also a strong cyclic stability. These were attributed to the intrinsic characteristics and morphology. The layered structure of $\mathrm{Zn}$-doped Ni-MOF with its enlarged inter- layer distance (Fig. 21b) ensured more efficient ion intercalation and deintercalation, thereby improving the pseudocapacitance.

Weng et al. [95] prepared a $\mathrm{MoS}_{2} /$ microporous carbon (MPC) composite by loading ZIF-8 on $\mathrm{MoS}_{2}$ nanosheets and subjecting this to further carbonization. The capacitance retention was $98 \%$ of the initial capacity after 3,000 cycles, while specific capacitance of $189 \mathrm{Fg}^{-1}$ was achieved in $1 \mathrm{~mol} \mathrm{~L}^{-1} \mathrm{H}_{2} \mathrm{SO}_{4}$ electrolyte at $1 \mathrm{~A} \mathrm{~g}^{-1}$. $\mathrm{MoS}_{2}$ wrapped with MPC promotes the cyclic stability, and gave rise to a new approach to building high performance supercapacitor electrodes.

\section{Carbon-based materials as additional materials}

Graphene and carbon nanotubes are widely investigated conductive fillers to the supercapacitor electrodes due to their superior electrical conductivity and large SSA $[96,97]$. Moreover, the two dimensional (2D) structure of graphene and one dimensional (1D) structure of carbon nanotubes can serve as the substrate due to the excellent mechanical and chemical stability.

Li et al. [98] prepared multi-wall carbon nanotubes (MWCNTs)/porous carbon material by carbonizing $\left(800^{\circ} \mathrm{C}\right)$ and acid treating the MWCNTs composites, which decorated the ZIF-8 onto the surface (Fig. 22). The MWCNTS was functionalized by $\mathrm{KMnO}_{4}$ and $\mathrm{HNO}_{3}$. The specific capacitance value of MWCNTs/nanoporous carbon-Large was measured to be $302.2 \mathrm{~F} \mathrm{~g}^{-1}$ in $1 \mathrm{~mol} \mathrm{~L}^{-1}$ $\mathrm{H}_{2} \mathrm{SO}_{4}$ electrolyte, at a current density of $2 \mathrm{~A} \mathrm{~g}^{-1}$. Furthermore, the loss of capacitance as measured by cyclic voltammetry, was only $4.2 \%$ after 10,000 cycles at a scan rate of $100 \mathrm{mV} \mathrm{s}^{-1}$. This strong cyclability indicates the MWCNTs/nanoporous carbon-Large may be a promising material for supercapacitor electrodes.

$\mathrm{Xu}$ et al. [99] also obtained hybrid carbon nanotubes (CNTs)/nitrogen-doped carbon polyhedra by carbonizing $\left(1000^{\circ} \mathrm{C}\right) \mathrm{CNTs} / \mathrm{ZIF}-8$, which come from in situ growth of ZIF-8 on the CNTs substrate. When the scanning rate was $5 \mathrm{mV} \mathrm{s}^{-1}$, the specific capacitance reached $308.0 \mathrm{~F} \mathrm{~g}^{-1}$ in $1 \mathrm{~mol} \mathrm{~L}^{-1} \mathrm{H}_{2} \mathrm{SO}_{4}$ electrolyte. Wen et al. [100] prepared $\mathrm{Ni}-\mathrm{MOF} / \mathrm{CNTs}$ by loading Ni-MOF on CNTs. Nickel chloride and $\mathrm{H}_{2} \mathrm{BDC}$ were used to prepare $\mathrm{Ni}-\mathrm{MOF}$. The specific capacitance of the as-prepared Ni-MOF/CNTs was $1,765 \mathrm{~F} \mathrm{~g}^{-1}$ in $6 \mathrm{~mol} \mathrm{~L}^{-1} \mathrm{KOH}$ electrolyte, which was greater than that of pristine $\mathrm{Ni}-\mathrm{MOF}\left(1,080 \mathrm{Fg}^{-1}\right)$ at $0.5 \mathrm{~A} \mathrm{~g}^{-1}$. The nanoporous structure of Ni-MOF can improve the penetration of the electrolyte, thereby increasing its contact area with electrochemically active sites. Meanwhile, the CNTs wrapped with Ni-MOF can improve the transport of electrons from the active compo- 
a

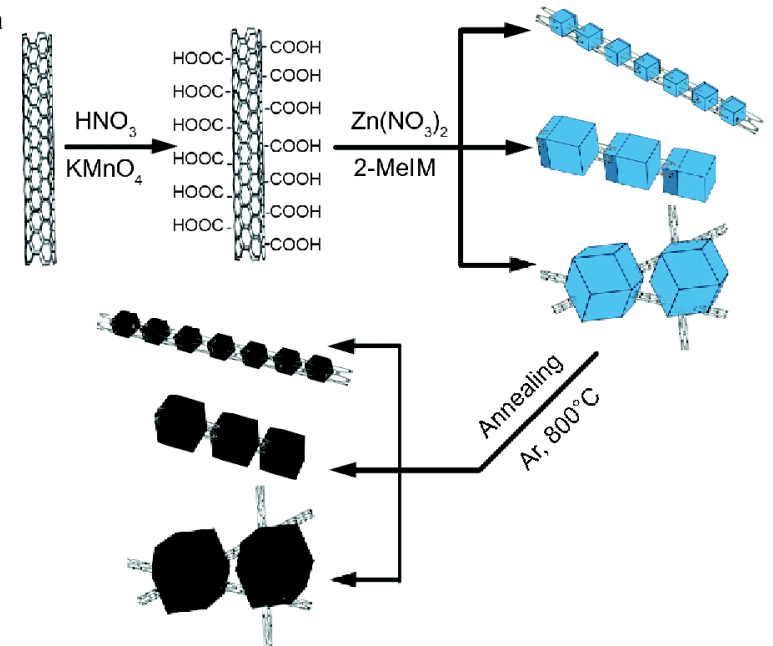

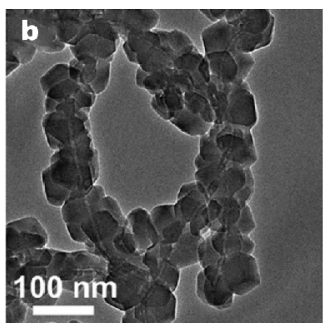
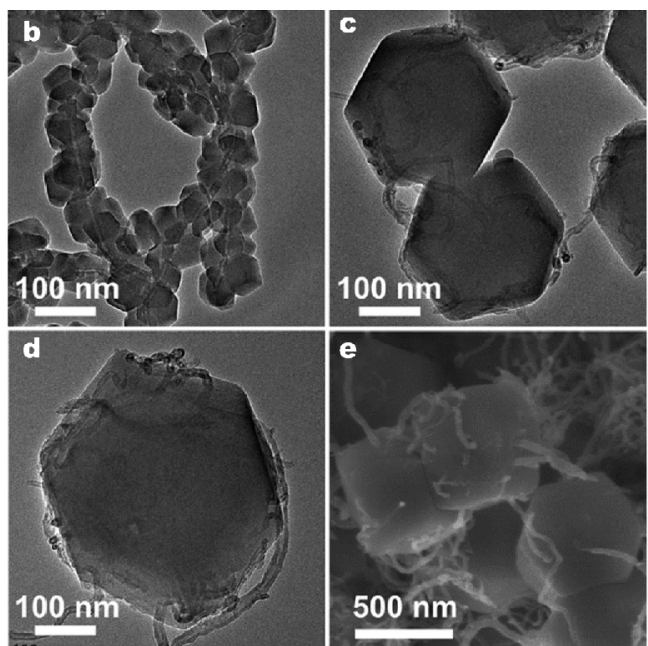

Figure 22 (a) Schematic for the fabrication process of MWCNTs/nanoporous carbon-Small/Middle/Large. (b, c, d) TEM images of MWCNTs/ nanoporous carbon with different sizes. (e) SEM image of MWCNTs/nanoporous carbon-Large. Reproduced with permission from Ref. [98], Copyright 2017, the Royal Society of Chemistry.

nent to the current collector during the charge/discharge process.

Zhang et al. [101] prepared CNTs/Mn-MOF by growing Mn-MOF on CNTs. The manganese acetate and ammonium phthalate $\left(\mathrm{NH}_{4}\right)_{2} \mathrm{C}_{8} \mathrm{H}_{4} \mathrm{O}_{4}$ were used to prepare Mn-MOF. The carboxylic groups on CNTs acted as nucleation centers for subsequent Mn-MOF loading. The specific capacitance was calculated to be $202.8 \mathrm{~F} \mathrm{~g}^{-1}$ in $1 \mathrm{~mol} \mathrm{~L}^{-1} \mathrm{Na}_{2} \mathrm{SO}_{4}$ electrolyte at $1 \mathrm{~A} \mathrm{~g}^{-1}$.

Zhang et al. [102] obtained ZnO QDs/carbon/CNT $\left(650^{\circ} \mathrm{C}\right)$ and $\mathrm{PNC} / \mathrm{CNTs}\left(1000^{\circ} \mathrm{C}\right)$ by thermal treatment of ZIF-8/CNTs. ZnO QDs/carbon/CNTs delivered a specific capacitance of $185 \mathrm{~F} \mathrm{~g}^{-1}$ at $0.5 \mathrm{~A} \mathrm{~g}^{-1}$ while $\mathrm{PNC} /$ carbon/CNTs displayed a specific capacitance of $250 \mathrm{~F} \mathrm{~g}^{-1}$ at $1 \mathrm{~A} \mathrm{~g}^{-1}$ in $1 \mathrm{~mol} \mathrm{~L}^{-1} \mathrm{Na}_{2} \mathrm{SO}_{4}$ electrolyte.

ZIF-8@reduced graphene oxides (RGO) was fabricated by Kim et al. [103] after annealing treatment of the ZIF-8/ graphene composites. They found that the higher annealing temperature, the larger SSA, and the higher the pore volume obtained. Zhou et al. [104] prepared NiMOF@GO through the reaction of GO together with nickel nitrate and PTA (Fig. 23a). Different temperatures and different weight ratios of GO and MOF were used to prepare Ni-MOF and Ni-MOF@GO. It was found that when the reaction temperature was $180^{\circ} \mathrm{C}$, in a three electrode configuration, the specific capacitance of $\mathrm{Ni}$ MOF-HCl-180 with a modulator of $\mathrm{HCl}$ reached $1,457.7 \mathrm{Fg}^{-1}$ in $2 \mathrm{~mol} \mathrm{~L}^{-1} \mathrm{KOH}$ electrolyte at $1 \mathrm{~A} \mathrm{~g}^{-1}$. Moreover, the capacitance retention ratio was $83.4 \%$ of the initial capacity after 3,000 cycles (Fig. 23c). A maximum specific capacitance of $2,192.4 \mathrm{~F} \mathrm{~g}^{-1}$ was measured with Ni-MOF@GO-3 of 3 wt.\% GO weight ratio in $2 \mathrm{~mol} \mathrm{~L}^{-1} \mathrm{KOH}$ electrolyte at $1 \mathrm{~A} \mathrm{~g}^{-1}$, together with a capacitance retention ratio of $85.1 \%$ after 3,000 cycles. The as-prepared Ni-MOF@GO exhibited excellent electrochemical performance (Fig. 23b, c), showing tremendous potential as a supercapacitor electrode material.

Yin et al. [105] synthesized RGO@ $\mathrm{Co}_{3} \mathrm{O}_{4}$ and $\mathrm{Co}_{3} \mathrm{O}_{4}$ RGO- $\mathrm{Co}_{3} \mathrm{O}_{4}$ by annealing GO@ZIF-67 and ZIF67@GO@ZIF-67, which was prepared using GO, cobalt nitrate, 2-methylimidazole (MeIm) and PVP (Fig. 24). In their work, a SSA of $198.4 \mathrm{~m}^{2} \mathrm{~g}^{-1}$ was achieved for RGO@ $\mathrm{Co}_{3} \mathrm{O}_{4}$, which was about 5 times greater than that of $\mathrm{Co}_{3} \mathrm{O}_{4}\left(32.54 \mathrm{~m}^{2} \mathrm{~g}^{-1}\right)$. The specific capacitance of

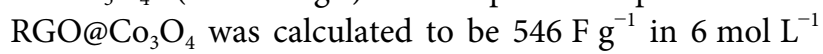
$\mathrm{KOH}$ electrolyte at $0.5 \mathrm{~A} \mathrm{~g}^{-1}$. The porous structure of this composite material can not only improve the permeability of the electrolyte, shortening the diffusion distance for ions, but also reduces the volume change of the electrode during the charge/discharge process. Furthermore, the higher surface area, as compared with the $\mathrm{Co}_{3} \mathrm{O}_{4}$-only films, offers increased contact area between electrolyte and electrode. In addition, the electrochemical performance of the composites films was enhanced due to the higher conductivity of the incorporated RGO.

\section{Additional materials after MOFs are prepared}

\section{Organic compounds as additional materials}

Wang et al. [106] reported a novel composite polyanilineZIF-67-carbon cloth (PANI-ZIF-67-CC) (Fig. 25). Beginning with carbon cloth, ZIF-67 was coated onto the 
a

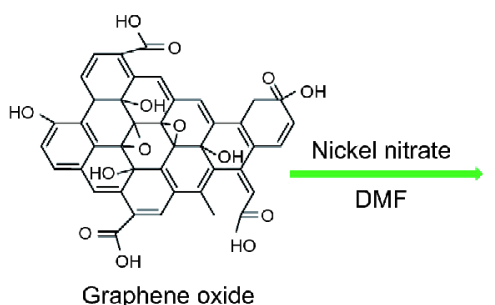

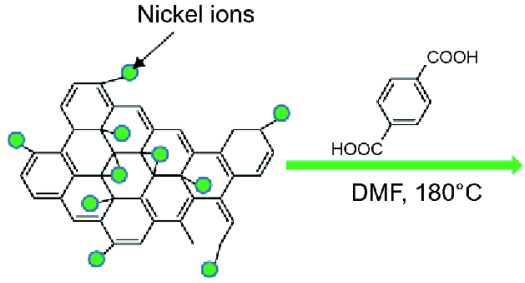

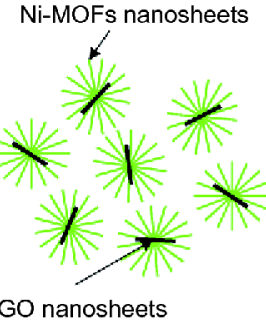

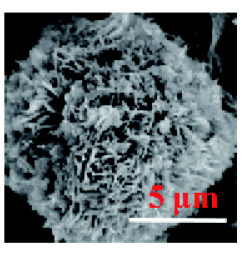
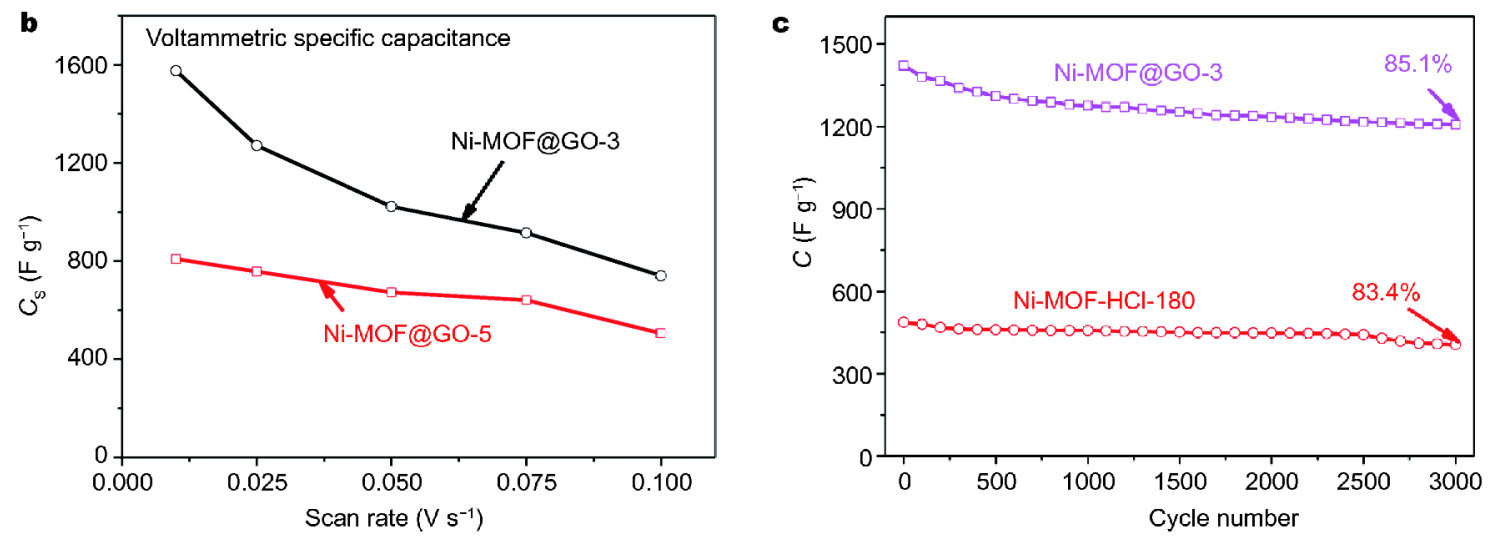

Figure 23 (a) Illustration of Ni-MOFs with graphene oxide (GO) nanosheets; (b) the specific capacitance of Ni-MOF@GO with different GO weight ratio; (c) cyclic stability of Ni-MOF-HCl-180 and Ni-MOF@GO-3. Reproduced with permission from Ref. [104], Copyright 2016, American Chemical Society.
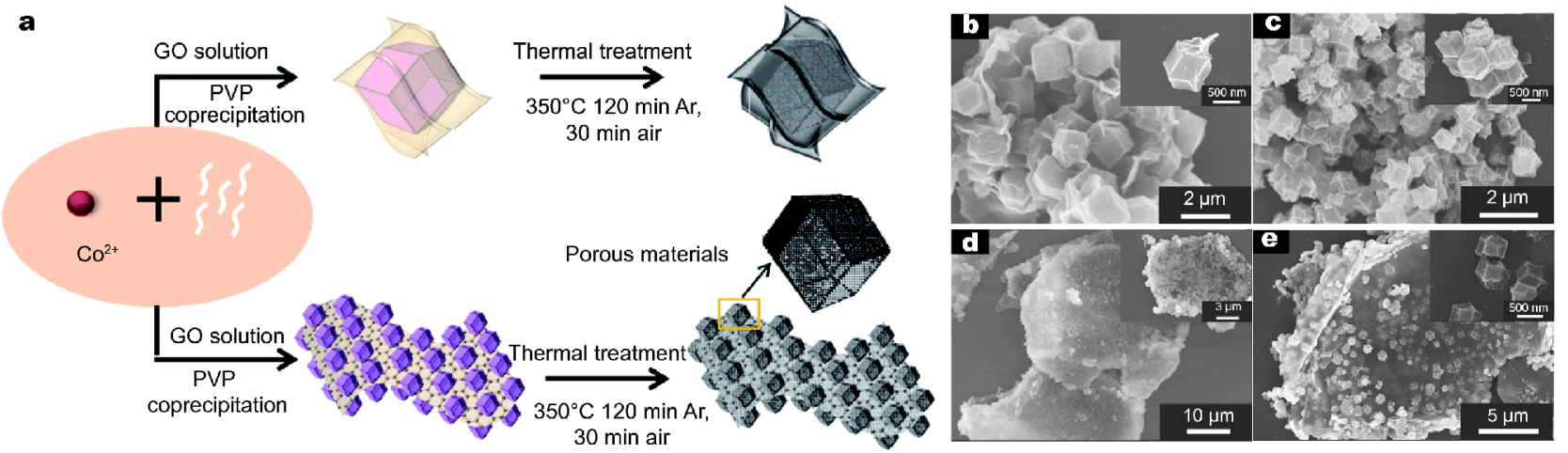

Figure 24 (a) Schematic illustration of the synthesis processes of $\mathrm{RGO} @ \mathrm{Co}_{3} \mathrm{O}_{4}$ and $\mathrm{Co}_{3} \mathrm{O}_{4}-\mathrm{RGO}-\mathrm{Co}_{3} \mathrm{O}_{4} \cdot \mathrm{SEM}$ images of GO@ZIF (b); $\mathrm{RGO@Co} \mathrm{O}_{4}$ (c); $\mathrm{ZIF} / \mathrm{GO} / \mathrm{ZIF}$ (d) and $\mathrm{Co}_{3} \mathrm{O}_{4}-\mathrm{RGO}-\mathrm{Co}_{3} \mathrm{O}_{4}$ (e). Reproduced with permission from Ref. [105], Copyright 2016, Elsevier.

cloth, and then following this, PANI was deposited by an electrochemical process [107]. In this study, carbon cloth serves as the substrate on which the active material is formed, and is able to be retained as the current collector for device construction. The advantages of this procedure are the cloth simplifies the production route, and further offers low cost and good flexibility. The chains of PANI can link up the isolated MOF and act as bridges for electrons transportation between the external circuit and the internal surface of MOFs (Fig. 25g). PANI-ZIF-67-CC exhibited a SSA of $73 \mathrm{~m}^{2} \mathrm{~g}^{-1}$ which was lower than that of ZIF-67. The areal capacitance of PANI-ZIF-67-CC was measured to be $2,146 \mathrm{mF} \mathrm{cm}^{-2}$ in $3 \mathrm{~mol} \mathrm{~L}^{-1} \mathrm{KCl}$ electrolyte at $10 \mathrm{mV} \mathrm{s}^{-1}$. Moreover, the electrical conductivity of the PANI-ZIF-67-CC was increased due to the deposition of PANI (Fig. 25g), resulting in enhanced rate performance.

Guo et al. [108] prepared carbonized Zn-MOF/PANI 

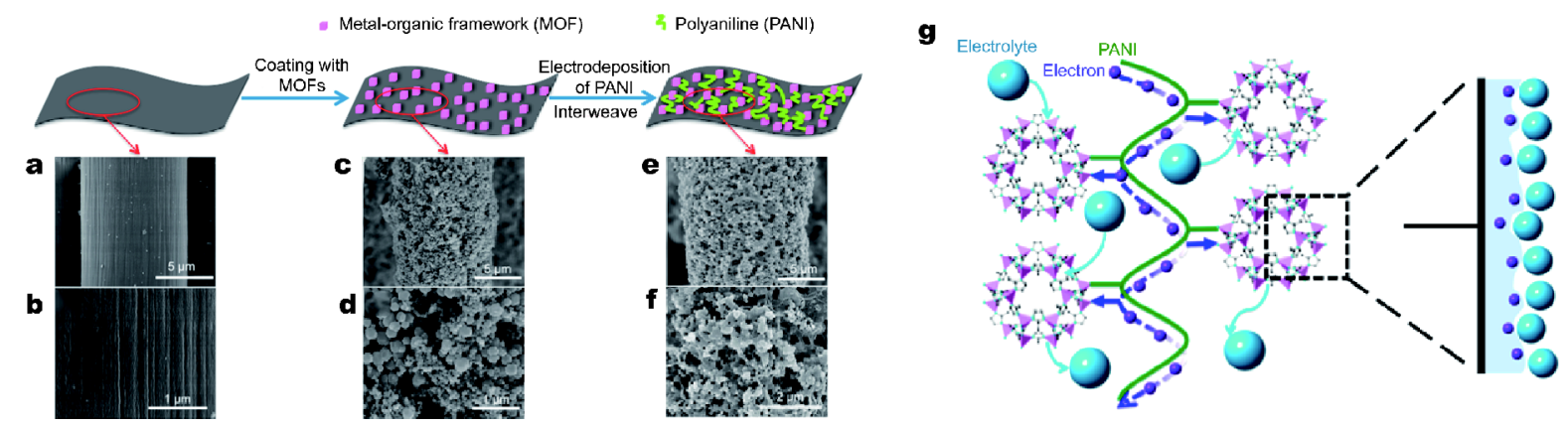

Figure 25 Schematic of the two-step fabrication of PANI-ZIF-67-CC and SEM images of (a, b) carbon cloths, ZIF-67-CC (c, d), and PANI-ZIF-67CC (e, f). (g) Electron and electrolyte can access MOF surfaces and form an EDLC on the surface of PANI-ZIF-67-CC. Reproduced with permission from Ref. [106], Copyright 2015, American Chemical Society.
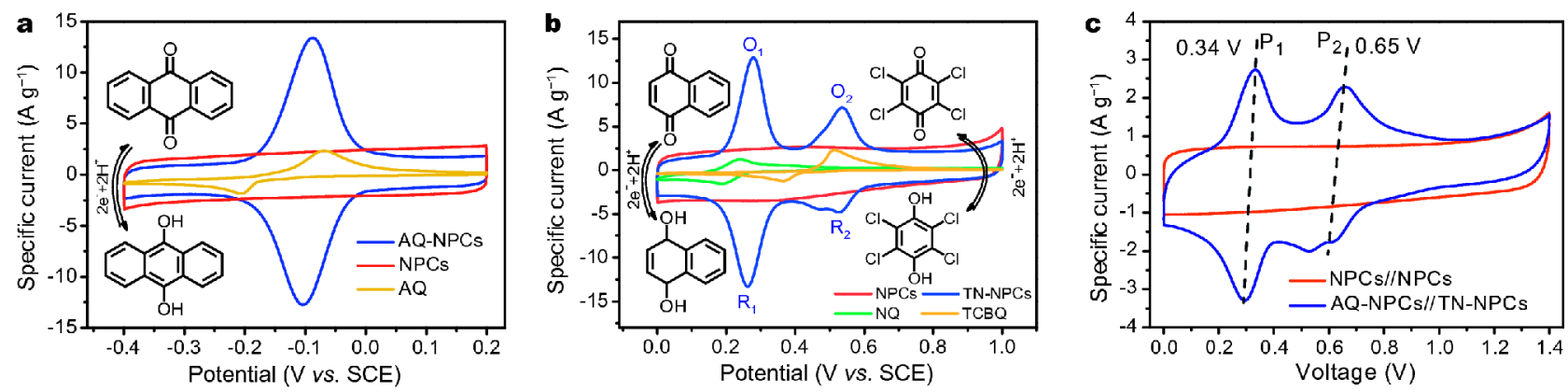

Figure 26 (a) CV curves of AQ-NPCs, pure NPCs and AQ at $10 \mathrm{mV} \mathrm{s}^{-1}$. (b) CV curves of TN-NPCs, pure NPCs, NQ and TCBQ at $10 \mathrm{mV} \mathrm{s}$. (c) The $\mathrm{CV}$ curves of ASC at $10 \mathrm{mV} \mathrm{s}^{-1}$. Reproduced with permission from Ref. [109], Copyright 2017, Elsevier.

(ZMP) via in situ chemical oxidative polymerization. Typically, carbonized Zn-MOF was obtained by using zinc nitrate, 8-hydroxyquinoline and subsequent annealing treatment. The addition of carbonized Zn-MOF enhanced the electrical conductivity of PANI and the stability of the structure. Furthermore, the layered structure of carbonized ZMP can reduce the transmission distance of electrons and diffusion resistance of electrolyte. Carbonized ZMP-0.04 with $0.04 \mathrm{~g}$ of MOF addition amount displayed a maximum specific capacitance of $477 \mathrm{~F} \mathrm{~g}^{-1}$ in $1 \mathrm{~mol} \mathrm{~L}^{-1} \mathrm{H}_{2} \mathrm{SO}_{4}$ electrolyte at $1 \mathrm{~A} \mathrm{~g}^{-1}$.

Guo et al. [109] developed a general strategy to fabricate nitrogen-doped porous carbon (NPCs) material for supercapacitor electrodes using ZIF-8 as a sacrificial template and modification of pure anthraquinone ( $\mathrm{AQ})$, mixed naphthoquinone (NQ) and tetrachlorobenzoquinone (TCBQ). The TCBQ\&NQ-NPCs (TN-NPCs) exhibited higher electrochemical activity than TCBQ and NQ. AQ-NPCs and TN-NPCs have good stability due to the $\pi-\pi$ stacking between electroactive organic compounds (AQ, TN) and NPCs. Furthermore, electroactive organic AQ, NQ and TCBQ in the $\mathrm{H}_{2} \mathrm{SO}_{4}$ electrolyte can participate in the redox reaction and then enhance the pseudocapacitance (Fig. 26). The SSA of as- prepared TN-NPCs was found to be $356 \mathrm{~m}^{2} \mathrm{~g}^{-1}$, which was higher than that of AQ-NPCs $\left(268 \mathrm{~m}^{2} \mathrm{~g}^{-1}\right)$. TN-NPCs showed a specific capacitance of $392 \mathrm{~F} \mathrm{~g}^{-1}$ in $1 \mathrm{~mol} \mathrm{~L}^{-1}$ $\mathrm{H}_{2} \mathrm{SO}_{4}$ electrolyte, which was higher than that of AQNPCs exhibiting $373 \mathrm{Fg}^{-1}$ at the current density of $1 \mathrm{~A} \mathrm{~g}^{-1}$. On the other hand, the rate capabilities of AQNPCs and TN-NPCs at $50 \mathrm{~A} \mathrm{~g}^{-1}$ were $83.2 \%$ and $76.9 \%$ with aspect to that at $1 \mathrm{~A} \mathrm{~g}^{-1}$, indicating that the pore diameter and higher conductivity had a positively impact on charge/discharge process. It was also found that the specific capacitance of AQ-NPCs was proportional to the scanning rate, which demonstrates that the AQ-NPCs are with a suitable pore structure. For the ASCs constructed in this study, AQ-NPCs were used as negative electrodes while TN-NPCs as positive electrodes. The specific capacitance value of AQ-NPCs//TN-NPCs supercapacitor was $86 \mathrm{~F} \mathrm{~g}^{-1}$ in $1 \mathrm{~mol} \mathrm{~L}^{-1} \mathrm{H}_{2} \mathrm{SO}_{4}$ electrolyte at $1 \mathrm{~A} \mathrm{~g}^{-1}$, together with $75.3 \%$ capacitance retention ratio after 5,000 cycles at $5 \mathrm{~A} \mathrm{~g}^{-1}$. The electrochemical performance of the supercapacitor benefited from the porous structure of the positive and negative electrodes, which can ensure enough electrolyte ions for EDLC and organic redox reactions. Furthermore, the redox reactions of the electroactive organic molecules in the electrode were not 

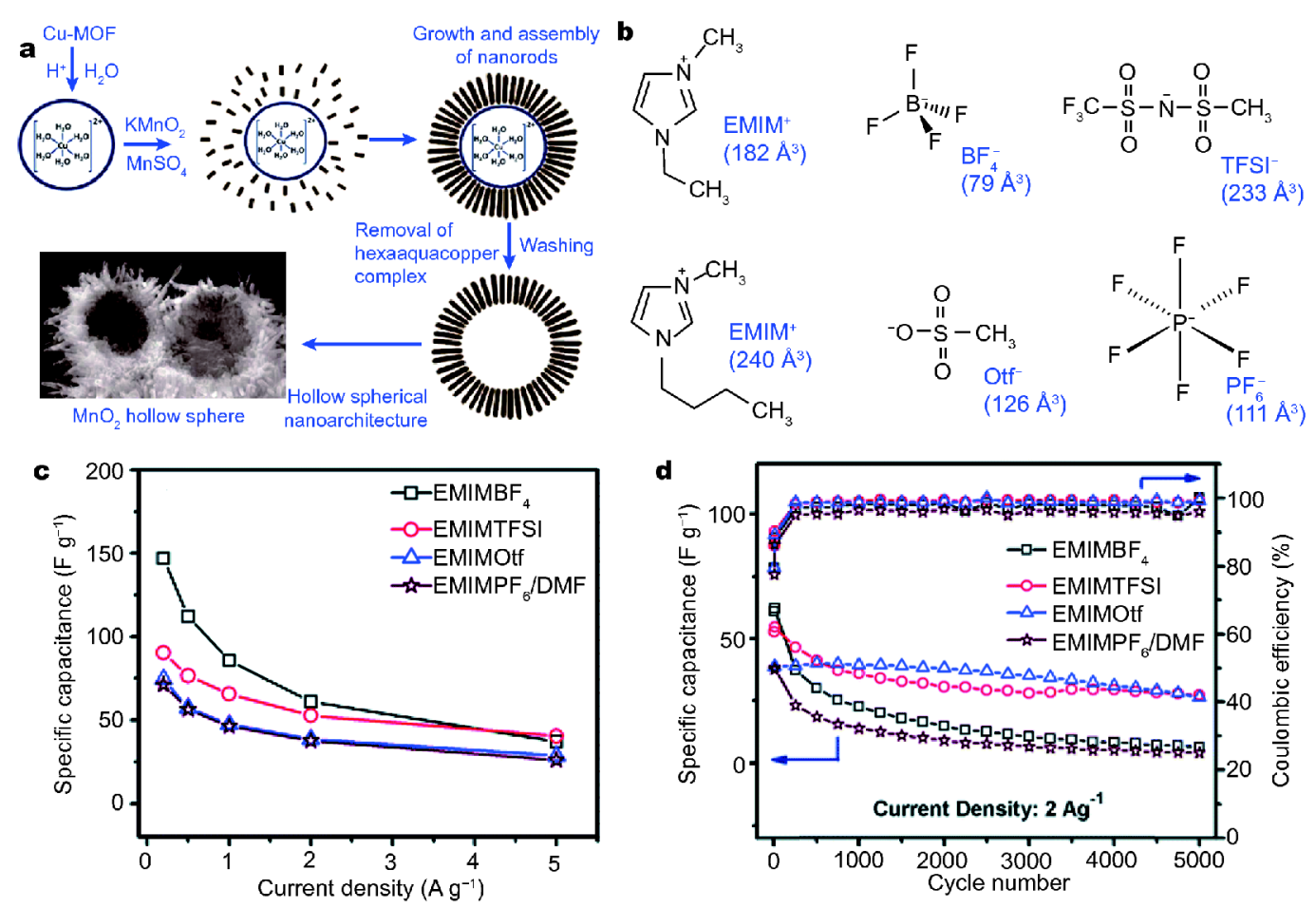

Figure 27 (a) The formation mechanism of $\mathrm{MnO}_{2}$ hollow spheres; (b) chemical structure representation of the constituent ions in the ionic liquid electrolytes; (c) the specific capacitance of $\mathrm{AC} / / \mathrm{IL} / / \mathrm{MnO}_{2} \mathrm{ASC}$ in different electrolytes; (d) cyclic stability of $\mathrm{AC} / / \mathrm{IL} / / \mathrm{MnO} \mathrm{ASC}_{2}$ in different electrolytes. Reproduced with permission from Ref. [110], Copyright 2015, the Royal Society of Chemistry.

controlled by the diffusion, resulting in a kinetic condition for the self-matching of positive and negative electrode half reactions.

Metals as additional materials

Maiti et al. [110] fabricated hollow spherical nanostructured $\mathrm{MnO}_{2}$ via low temperature solvothermal method under acidic conditions (Fig. 27a). The hollow spherical nanostructured $\mathrm{MnO}_{2}$ was formed by the unstable metal ligand bonding in $\mathrm{Cu}-\mathrm{BTC}$. The $\mathrm{Cu}^{2+}$ and the water molecules are coordinated to act as nucleation sites in the acidic conditions, which is a key part. The SSA of $\mathrm{MnO}_{2}$ was measured to be $85 \mathrm{~m}^{2} \mathrm{~g}^{-1}$, with a $16 \mathrm{~nm}$ diameter pore mode. It is proposed that the smaller electrolyte anions achieves contact on a greater surface area of the electrode material, resulting in a higher EDLC (Fig. 27b). Therefore, the superior electrochemical performance with a specific capacitance of $147 \mathrm{~F} \mathrm{~g}^{-1}$ at $0.2 \mathrm{~A} \mathrm{~g}^{-1}$ was measured in the 1-ethyl-3-methylimidazolium tetrafluoroborate (EMIMBF) electrolyte (Fig. 27c, d).

Jiang et al. [111] firstly synthesized the layered double hydroxides (LDHs) non-spherical structures by ZIF-67 nanocrystals as templates. The Ni-Co nanocages well inherited the rhombic dodecahedral shape of the ZIF-67 templates, and the shell was composed of nanosheets assembled with an edge-to-face stacking. Specifically, a hollow structure was obtained after precipitation of the corresponding shell materials and acidic etching of the sacrificial template. In order to form nanocages structure, the reaction kinetics needs to keep balance between the precipitation of the shell and the simultaneous template etching. The Ni-Co LDH nanocages delivered a superior specific capacitance of $1,203 \mathrm{~F} \mathrm{~g}^{-1}$ at $1 \mathrm{~A} \mathrm{~g}^{-1}$ in $1 \mathrm{~mol} \mathrm{~L}^{-1}$ $\mathrm{KOH}$ electrolyte than Ni-Co LDH nanoparticles, together with excellent cycling stability (i.e., $90.2 \%$ of the initial capacity retained after 1000 cycles). However, the poor conductivity affects the overall performance of pseudocapacitance by reducing the energy efficiency.

\section{Carbon-based materials as additional materials}

Srimuk et al. [112] prepared a flexible electrode material using carbon fiber paper, which was spin-coated with the Hong Kong University of Science and Technology-1 (HKUST-1) Cu-MOF and RGO. The SSA of $10 \mathrm{wt} . \%$ RGO/HKUST-1 was $1,241 \mathrm{~m}^{2} \mathrm{~g}^{-1}$ and the average pore diameter was $8.2 \mathrm{~nm}$. The specific capacitance of the constructed flexible electrode was measured to be $385 \mathrm{~F} \mathrm{~g}^{-1}$ at the current density of $1 \mathrm{~A} \mathrm{~g}^{-1}$ in $0.5 \mathrm{~mol} \mathrm{~L}^{-1}$ 

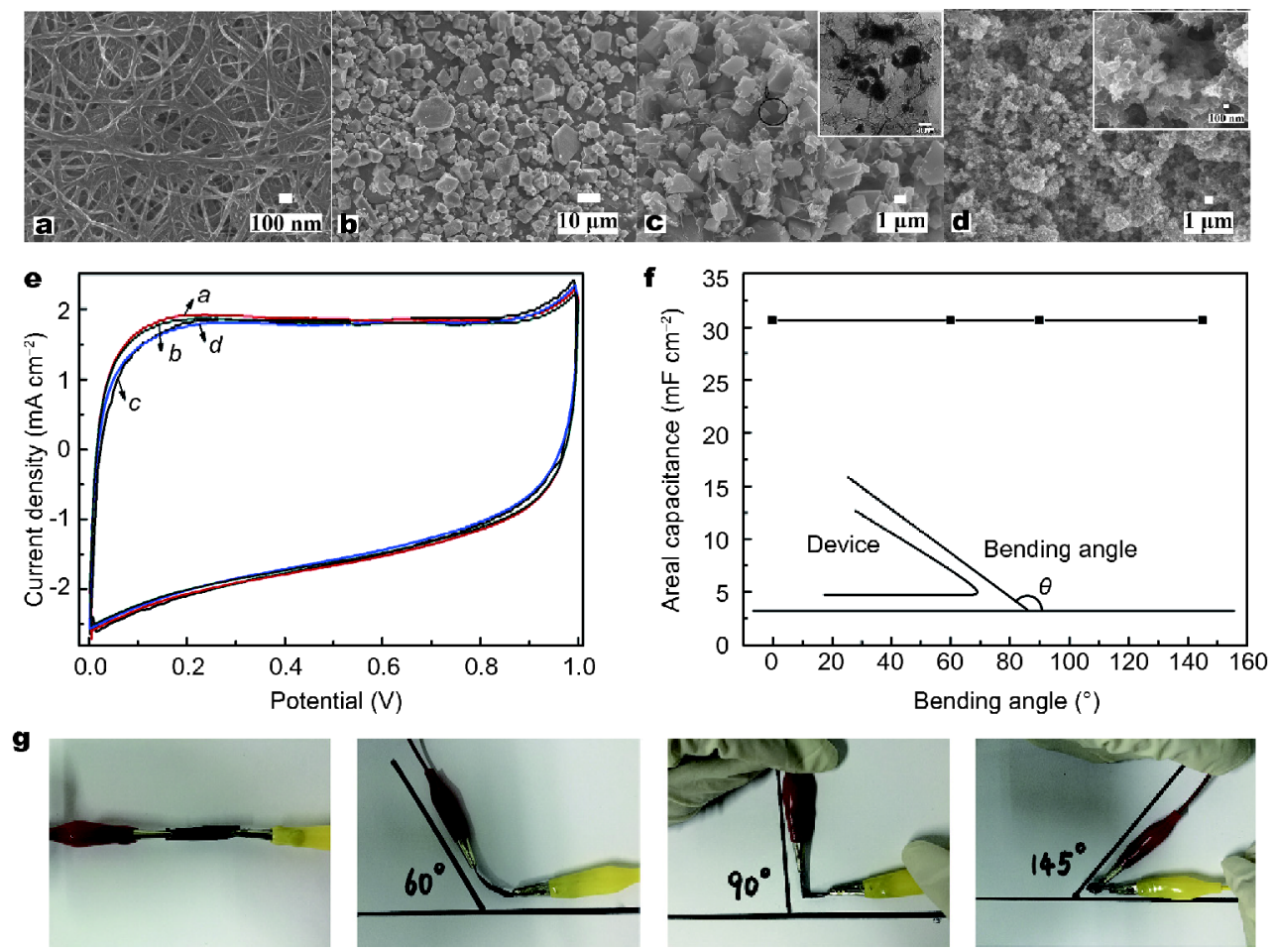

Figure 28 SEM images of carbon nanotube film (CNTF) (a) and HKUST-1 (b); (c) SEM image of H-1-15G, and the inset is a high resolution image; (d) SEM image of PEDOT/H-15G-CNTF, and the inset is a high resolution image; (e) the CV curves at $50 \mathrm{mV} \mathrm{s}^{-1}$ for solid-state supercapacitors bent at different angles; (f) the specific capacitance of the device bent at different angels and (g) the accompanying photographs. Reproduced with permission from Ref. [113], Copyright 2016, Elsevier.

$\mathrm{Na}_{2} \mathrm{SO}_{4}$ electrolyte.

$\mathrm{Fu}$ et al. [113] synthesized a novel electrode material using poly(3,4-ethylenedioxythiophene (PEDOT). Firstly, HKUST-1 was prepared by solvothermal method. Then, a GO solution was added to the well-dissolved HKUST-1 precursors forming H-G hybrid materials. H-G hybrid materials were then loaded on carbon nanotube film (CNTF) to obtain H-G-CNTF. Finally, PEDOT/H-GCNTF was prepared by electrochemical deposition of the PEDOT onto the H-G-CNTF (Fig. 28). A maximum SSA of $950 \mathrm{~m}^{2} \mathrm{~g}^{-1}$ was achieved for $\mathrm{H}-15 \mathrm{G}$, with an accompanying $5.5 \mathrm{~nm}$ average pore diameter. The PEDOT/H15G-CNTF electrode showed an areal capacitance of $128 \mathrm{mF} \mathrm{cm}^{-1}$ in $3 \mathrm{~mol} \mathrm{~L}^{-1} \mathrm{KCl}$ electrolyte at $10 \mathrm{mV} \mathrm{s}^{-1}$. In addition, an all solid-state supercapacitor was assembled to further study the electrochemical properties employing $\mathrm{PVA} / \mathrm{H}_{3} \mathrm{PO}_{4}$ as the gel electrolyte. A specific capacitance of $37.8 \mathrm{mF} \mathrm{cm}^{-2}$ was measured for the supercapacitor device at a scan rate of $5 \mathrm{mV} \mathrm{s}^{-1}$ (Fig. 28e). It is worth noting that the capacitance did not decrease under various bending conditions the supercapacitor device was subjected to (Fig. 28f, g).

Banerjee et al. [114] synthesized MOF- $5_{\text {NiRGO }}$ in bulk (gram scale) quantities. The composites were prepared by mixing Ni-doped MOF-5 with RGO (Fig. 29). The asprepared MOF- $5_{\mathrm{Ni} 50 \%}$ RGO50\% exhibited a specific capacitance of $758 \mathrm{~F} \mathrm{~g}^{-1}$ in $1 \mathrm{~mol} \mathrm{~L}^{-1} \mathrm{KOH}$ electrolyte at $1 \mathrm{mV} \mathrm{s}^{-1}$, which was much greater than that of MOF$5_{\mathrm{Ni} 50 \%}\left(380 \mathrm{~F} \mathrm{~g}^{-1}\right)$ and MOF-5 $\left(90 \mathrm{~F} \mathrm{~g}^{-1}\right)$. Other than increasing the contact area between the electrode and electrolyte, the addition of RGO also enhanced the conductivity of the electrode, thereby promoting the efficiency of ion transport, resulting in higher capacitive performance.

\section{CONCLUSION AND FUTURE PERSPECTIVES}

In summary, MOF materials have attracted extensive attention in response to an ever increasing demand for high performance supercapacitor electrodes. In efforts to meet the criteria of the adjustable pore size distribution, high SSA, abundant active sites and excellent electrical conductivity of electrode, various nanoscale MOFs structures and their derivatives have been intensively explored. Currently, MOFs represent the strong candidate for supercapacitor electrodes due to their ordered porous 


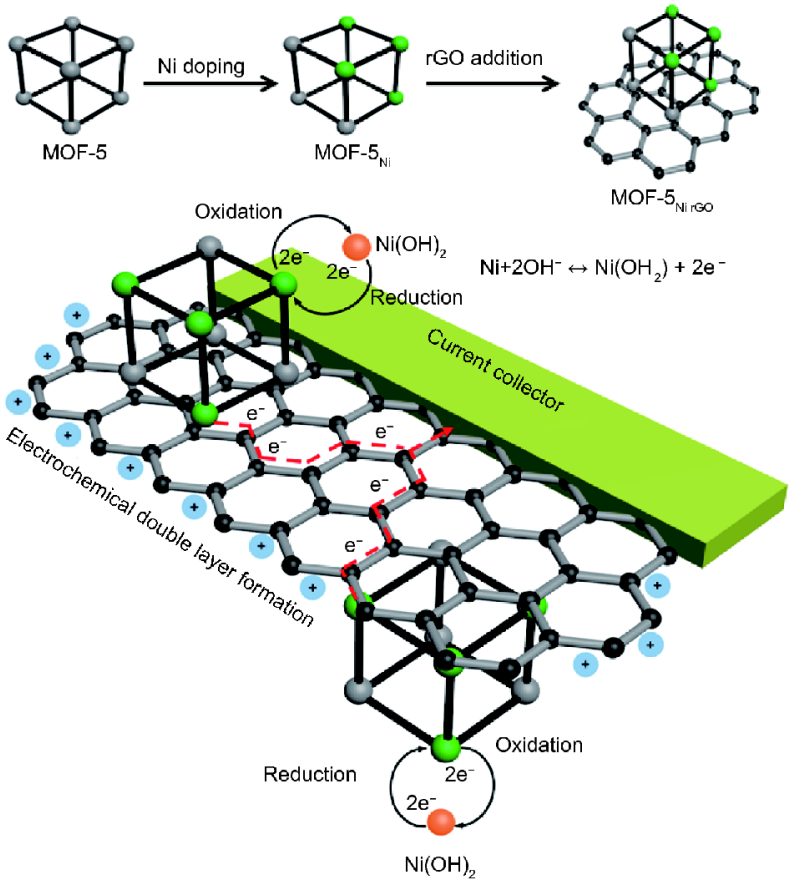

Figure 29 Schematic of the fabrication of MOF- $5_{\text {NiRGO }}$ applied as a supercapacitor electrode, and also depicted are the coexisting EDLC charge storage RGO, and the reversible redox reactions of $\mathrm{Ni}$ metal centers. Reproduced with permission from Ref. [114], Copyright 2016, American Chemical Society.

structure combined with reversible intercalation of electrolyte ions. However, it is more effective to enhance capacitance by introducing pseudocapacitive materials into the MOF systems with porous structure. Although transition metal oxides are widely used as active materials to improve the capacitance due to their extremely high theoretical capacitance, the Faradaic reaction takes time to store charges at a level of full capacity of the electrode, and such pseudocapacitive electrodes suffer from low rate performance. Moreover, most active electrodes of transition metal oxides suffer from lower structural/chemical stability which greatly limits the cycle life of a supercapacitor. Coating carbon material onto MOFs and constructing double shell structure are common strategies to reduce the irreversible capacity loss caused in charge/ discharge processes. The numerous examples of this demonstrate that rational architecture of MOFs materials, through combining carbon-based materials with polymer materials is an effective method to construct supercapacitor electrodes with the advantages of high capacity and long cycle life, making them promising for practical applications. Although most MOFs lack sufficient electrical conductivity, introducing materials with good electrical conductivity in MOFs system can endow fast dynamic charge propagation, thereby enhancing the electrochemical performance. Thus, it is of great significance to investigate the nature of charge transport in these materials, and to design suitable conductive structural MOFs.

A wide potential window is often required to boost the energy of a supercapacitor. However, the obstacle in using aqueous electrolyte is the electrolysis of water when the voltage exceeds $1.23 \mathrm{~V}$. The research of MOFs as supercapacitor electrodes is still concentrated at aqueous media and in the laboratory scale. Thus, more in-depth studies on solid-state supercapacitors with wider potential window are in need. Energy storage devices based on environmentally-friendly, low-cost and high-yield MOFs are anticipated to be commercially available in the future, based on the maturity of MOFs technology.

Received 21 September 2017; accepted 27 October 2017; published online 12 January 2018

1 Ozkan S, Nguyen NT, Hwang I, et al. Highly conducting spaced $\mathrm{TiO}_{2}$ nanotubes enable defined conformal coating with nanocrystalline $\mathrm{Nb}_{2} \mathrm{O}_{5}$ and high performance supercapacitor applications. Small, 2017, 13: 1603821

2 Shi P, Li L, Hua L, et al. Design of amorphous manganese oxide@multiwalled carbon nanotube fiber for robust solid-state supercapacitor. ACS Nano, 2017, 11: 444-452

3 Wang S, Liu N, Su J, et al. Highly stretchable and self-healable supercapacitor with reduced graphene oxide based fiber springs. ACS Nano, 2017, 11: 2066-2074

4 González-Gaitán C, Ruiz-Rosas R, Nishihara H, et al. Successful functionalization of superporous zeolite templated carbon using aminobenzene acids and electrochemical methods. Carbon, 2016, 99: $157-166$

5 Li X, Zhao Y, Bai Y, et al. A non-woven network of porous nitrogen-doping carbon nanofibers as a binder-free electrode for supercapacitors. Electrochim Acta, 2017, 230: 445-453

6 Dong L, Xu C, Li Y, et al. Flexible electrodes and supercapacitors for wearable energy storage: a review by category. J Mater Chem A, 2016, 4: 4659-4685

7 Huang C, Zhang J, Young NP, et al. Solid-state supercapacitors with rationally designed heterogeneous electrodes fabricated by large area spray processing for wearable energy storage applications. Sci Rep, 2016, 6: 25684

8 Wang Y, Shi Z, Huang Y, et al. Supercapacitor devices based on graphene materials. J Phys Chem C, 2009, 113: 13103-13107

9 Deka BK, Hazarika A, Kim J, et al. Recent development and challenges of multifunctional structural supercapacitors for automotive industries. Int J Energy Res, 2017, 41: 1397-1411

10 González A, Goikolea E, Barrena JA, et al. Review on supercapacitors: technologies and materials. Renew Sustain Energy Rev, 2016, 58: 1189-1206

$11 \mathrm{Ma} \mathrm{W}$, Chen S, Yang S, et al. Hierarchical $\mathrm{MnO}_{2}$ nanowire/graphene hybrid fibers with excellent electrochemical performance for flexible solid-state supercapacitors. J Power Sources, 2016, 306: 481-488

12 Wang D, Fang G, Xue T, et al. A melt route for the synthesis of 
activated carbon derived from carton box for high performance symmetric supercapacitor applications. J Power Sources, 2016, 307: 401-409

13 Wu S, Zhu Y. Highly densified carbon electrode materials towards practical supercapacitor devices. Sci China Mater, 2017, 60: 25-38

14 Zheng M, Xiao X, Li L, et al. Hierarchically nanostructured transition metal oxides for supercapacitors. Sci China Mater, 2017

15 Zhang Y, Zhen Z, Zhang Z, et al. In-situ synthesis of carbon nanotube/graphene composite sponge and its application as compressible supercapacitor electrode. Electrochim Acta, 2015, 157: 134-141

16 Sahu V, Shekhar S, Sharma RK, et al. Ultrahigh performance supercapacitor from lacey reduced graphene oxide nanoribbons. ACS Appl Mater Interfaces, 2015, 7: 3110-3116

17 Wang JG, Kang F, Wei B. Engineering of $\mathrm{MnO}_{2}$-based nanocomposites for high-performance supercapacitors. Prog Mater Sci, 2015, 74: 51-124

18 Li L, Song H, Zhang Q, et al. Effect of compounding process on the structure and electrochemical properties of ordered mesoporous carbon/polyaniline composites as electrodes for supercapacitors. J Power Sources, 2009, 187: 268-274

19 Liu M, Du Y, Miao YE, et al. Anisotropic conductive films based on highly aligned polyimide fibers containing hybrid materials of graphene nanoribbons and carbon nanotubes. Nanoscale, 2015, 7: 1037-1046

20 Wang K, Zhang X, Sun X, et al. Conducting polymer hydrogel materials for high-performance flexible solid-state supercapacitors. Sci China Mater, 2016, 59: 412-420

21 Belin T, Epron F. Characterization methods of carbon nanotubes: a review. Mater Sci Eng-B, 2005, 119: 105-118

$22 \mathrm{He} \mathrm{S}$, Chen W. Application of biomass-derived flexible carbon cloth coated with $\mathrm{MnO}_{2}$ nanosheets in supercapacitors. J Power Sources, 2015, 294: 150-158

23 Dhawale DS, Kim S, Park DH, et al. Hierarchically ordered porous $\mathrm{CoOOH}$ thin-film electrodes for high-performance supercapacitors. ChemElectroChem, 2015, 2: 497-502

$24 \mathrm{Wu} \mathrm{Q}, \mathrm{Xu} \mathrm{Y}$, Yao Z, et al. Supercapacitors based on flexible graphene/polyaniline nanofiber composite films. ACS Nano, 2010, 4: 1963-1970

25 Li ZF, Zhang H, Liu Q, et al. Fabrication of high-surface-area graphene/polyaniline nanocomposites and their application in supercapacitors. ACS Appl Mater Interfaces, 2013, 5: 2685-2691

26 Wang T, Zhang SL, Wang HX. Binary NiCu layered double hydroxide nanosheets for enhanced energy storage performance as supercapacitor electrode. Sci China Mater, 2017, doi: 10.1007/ s40843-017-9131-7

27 Nie Z, Wang Y, Zhang Y, et al. Multi-shelled $\alpha-\mathrm{Fe}_{2} \mathrm{O}_{3}$ microspheres for high-rate supercapacitors. Sci China Mater, 2016, 59: 247-253

28 Wang R, Luo Y, Chen Z, et al. The effect of loading density of nickel-cobalt sulfide arrays on their cyclic stability and rate performance for supercapacitors. Sci China Mater, 2016, 59: 629-638

29 Tran C, Kalra V. Co-continuous nanoscale assembly of nafionpolyacrylonitrile blends within nanofibers: a facile route to fabrication of porous nanofibers. Soft Matter, 2013, 9: 846-852

30 Choi KM, Jeong HM, Park JH, et al. Supercapacitors of nanocrystalline metal-organic frameworks. ACS Nano, 2014, 8: 74517457

31 Sun L, Campbell MG, Dincă M. Electrically conductive porous metal-organic frameworks. Angew Chem Int Ed, 2016, 55: 35663579

32 Zhang YZ, Cheng T, Wang Y, et al. A simple approach to boost capacitance: flexible supercapacitors based on manganese oxides@MOFs via chemically induced in situ self-transformation. Adv Mater, 2016, 28: 5242-5248

33 Stallinga P. Electronic transport in organic materials: comparison of band theory with percolation/(variable range) hopping theory. Adv Mater, 2011, 23: 3356-3362

34 Zhang Z, Yoshikawa H, Awaga K. Discovery of a "bipolar charging" mechanism in the solid-state electrochemical process of a flexible metal-organic framework. Chem Mater, 2016, 28: 12981303

35 Wei T, Zhang M, Wu P, et al. POM-based metal-organic framework/reduced graphene oxide nanocomposites with hybrid behavior of battery-supercapacitor for superior lithium storage. Nano Energy, 2017, 34: 205-214

36 Guan C, Zhao W, Hu Y, et al. Cobalt oxide and N-doped carbon nanosheets derived from a single two-dimensional metal-organic framework precursor and their application in flexible asymmetric supercapacitors. Nanoscale Horiz, 2017, 2: 99-105

37 Wang H, Gao Q, Hu J. Asymmetric capacitor based on superior porous $\mathrm{Ni}-\mathrm{Zn}-\mathrm{Co}$ oxide/hydroxide and carbon electrodes. J Power Sources, 2010, 195: 3017-3024

38 Yan X, Li X, Yan Z, et al. Porous carbons prepared by direct carbonization of MOFs for supercapacitors. Appl Surf Sci, 2014, 308: 306-310

39 Ding B, Wang J, Chang Z, et al. Self-sacrificial template-directed synthesis of metal-organic framework-derived porous carbon for energy-storage devices. ChemElectroChem, 2016, 3: 668-674

40 Mao ML, Sun LX, Xu F. Metal-organic frameworks/carboxyl graphene derived porous carbon as a promising supercapacitor electrode material. Key Eng Mater, 2017, 727: 756-763

41 Yi H, Wang $\mathrm{H}$, Jing $\mathrm{Y}$, et al. Asymmetric supercapacitors based on carbon nanotubes@NiO ultrathin nanosheets core-shell composites and MOF-derived porous carbon polyhedrons with super-long cycle life. J Power Sources, 2015, 285: 281-290

42 Meng JP, Gong Y, Lin Q, et al. Metal-organic frameworks based on rigid ligands as separator membranes in supercapacitor. Dalton Trans, 2015, 44: 5407-5416

43 Park SS, Hontz ER, Sun L, et al. Cation-dependent intrinsic electrical conductivity in isostructural tetrathiafulvalene-based microporous metal-organic frameworks. J Am Chem Soc, 2015, 137: $1774-1777$

44 Ye G, Gong Y, Keyshar K, et al. 3D reduced graphene oxide coated $\mathrm{V}_{2} \mathrm{O}_{5}$ nanoribbon scaffolds for high-capacity supercapacitor electrodes. Part Part Syst Charact, 2015, 32: 817-821

45 Lee DY, Yoon SJ, Shrestha NK, et al. Unusual energy storage and charge retention in Co-based metal-organic-frameworks. Micropor Mesopor Mater, 2012, 153: 163-165

46 Kandalkar SG, Dhawale DS, Kim CK, et al. Chemical synthesis of cobalt oxide thin film electrode for supercapacitor application. Synth Met, 2010, 160: 1299-1302

47 Zhang F, Hao L, Zhang LJ, et al. Solid-state thermolysis preparation of $\mathrm{Co}_{3} \mathrm{O}_{4}$ nano/micro superstructures from metal-organic framework for supercapacitors. Int J Electrochem Sci, 2011, 6: $2943-2954$

48 Lee DY, Shinde DV, Kim EK, et al. Supercapacitive property of metal-organic-frameworks with different pore dimensions and morphology. Micropor Mesopor Mater, 2013, 171: 53-57 
49 Miles DO, Jiang D, Burrows AD, et al. Conformal transformation of $[\mathrm{Co}(\mathrm{bdc})(\mathrm{DMF})]$ (Co-MOF-71, bdc $=1,4$-benzenedicarboxylate, $\mathrm{DMF}=\mathrm{N}, \mathrm{N}$-dimethylformamide) into porous electrochemically active cobalt hydroxide. Electrochem Commun, 2013, 27: 9-13

50 Yang J, Xiong P, Zheng C, et al. Metal-organic frameworks: a new promising class of materials for a high performance supercapacitor electrode. J Mater Chem A, 2014, 2: 16640-16644

51 Yan Y, Gu P, Zheng S, et al. Facile synthesis of an accordion-like Ni-MOF superstructure for high-performance flexible supercapacitors. J Mater Chem A, 2016, 4: 19078-19085

52 Lai F, Huang Y, Miao YE, et al. Controllable preparation of multidimensional hybrid materials of nickel-cobalt layered double hydroxide nanorods/nanosheets on electrospun carbon nanofibers for high-performance supercapacitors. Electrochim Acta, 2015, 174: 456-463

53 Jiao Y, Pei J, Yan C, et al. Layered nickel metal-organic framework for high performance alkaline battery-supercapacitor hybrid devices. J Mater Chem A, 2016, 4: 13344-13351

54 Xu J, Yang C, Xue Y, et al. Facile synthesis of novel metal-organic nickel hydroxide nanorods for high performance supercapacitor. Electrochim Acta, 2016, 211: 595-602

55 Worrall SD, Mann H, Rogers A, et al. Electrochemical deposition of zeolitic imidazolate framework electrode coatings for supercapacitor electrodes. Electrochim Acta, 2016, 197: 228-240

56 Yang J, Ma Z, Gao W, et al. Layered structural Co-based MOF with conductive network frames as a new supercapacitor electrode. Chem Eur J, 2017, 23: 631-636

57 Sheberla D, Bachman JC, Elias JS, et al. Conductive MOF electrodes for stable supercapacitors with high areal capacitance. Nat Mater, 2016, 16: 220-224

58 Pilon L, Wang H, d'Entremont A. Recent advances in continuum modeling of interfacial and transport phenomena in electric double layer capacitors. J Electrochem Soc, 2015, 162: A5158A5178

59 Jeon JW, Sharma R, Meduri P, et al. In situ one-step synthesis of hierarchical nitrogen-doped porous carbon for high-performance supercapacitors. ACS Appl Mater Interfaces, 2014, 6: 7214-7222

60 Hao F, Li L, Zhang X, et al. Synthesis and electrochemical capacitive properties of nitrogen-doped porous carbon micropolyhedra by direct carbonization of zeolitic imidazolate framework-11. Mater Res Bull, 2015, 66: 88-95

61 Yang Y, Hao S, Zhao H, et al. Hierarchically porous carbons derived from nonporous metal-organic frameworks: synthesis and influence of struts. Electrochim Acta, 2015, 180: 651-657

$62 \mathrm{Yu} \mathrm{M}$, Zhang L, He X, et al. 3D interconnected porous carbons from MOF-5 for supercapacitors. Mater Lett, 2016, 172: 81-84

63 Xia W, Qiu B, Xia D, et al. Facile preparation of hierarchically porous carbons from metal-organic gels and their application in energy storage. Sci Rep, 2013, 3: 1935

64 Mahmood A, Zou R, Wang Q, et al. Nanostructured electrode materials derived from metal-organic framework xerogels for high-energy-density asymmetric supercapacitor. ACS Appl Mater Interfaces, 2016, 8: 2148-2157

65 Tran C, Kalra V. Fabrication of porous carbon nanofibers with adjustable pore sizes as electrodes for supercapacitors. J Power Sources, 2013, 235: 289-296

66 Liu B, Shioyama H, Jiang H, et al. Metal-organic framework (MOF) as a template for syntheses of nanoporous carbons as electrode materials for supercapacitor. Carbon, 2010, 48: 456-463
67 Jin S, Deng H, Zhan L, et al. Synthesis of 3D hierarchical porous carbon as electrode material for electric double layer capacitors. New Carbon Mater, 2012, 27: 87-92

68 Lei Y, Gan M, Ma L, et al. Synthesis of nitrogen-doped porous carbon from zeolitic imidazolate framework-67 and phenolic resin for high performance supercapacitors. Ceram Int, 2017, 43: 6502-6510

69 Zhang H, Chen Y, Wang W, et al. Hierarchical Mo-decorated $\mathrm{Co}_{3} \mathrm{O}_{4}$ nanowire arrays on $\mathrm{Ni}$ foam substrates for advanced electrochemical capacitors. J Mater Chem A, 2013, 1: 8593

70 Guo Y, Yu L, Wang CY, et al. Hierarchical tubular structures composed of Mn-based mixed metal oxide nanoflakes with enhanced electrochemical properties. Adv Funct Mater, 2015, 25: 5184-5189

71 Liu X, Shi C, Zhai C, et al. Cobalt-based layered metal-organic framework as an ultrahigh capacity supercapacitor electrode material. ACS Appl Mater Interfaces, 2016, 8: 4585-4591

72 Yuan $\mathrm{C}, \mathrm{Wu} \mathrm{HB}, \mathrm{Xie} \mathrm{Y}$, et al. Mixed transition-metal oxides: design, synthesis, and energy-related applications. Angew Chem Int Ed, 2014, 53: 1488-1504

73 Yin Z, Chen Y, Zhao Y, et al. Hierarchical nanosheet-based Co$\mathrm{MoO}_{4}-\mathrm{NiMoO}_{4}$ nanotubes for applications in asymmetric supercapacitors and the oxygen evolution reaction. J Mater Chem A, 2015, 3: 22750-22758

74 Xie L, Hu Z, Lv C, et al. $\mathrm{Co}_{x} \mathrm{Ni}_{1-x}$ double hydroxide nanoparticles with ultrahigh specific capacitances as supercapacitor electrode materials. Electrochim Acta, 2012, 78: 205-211

75 Liang D, Tian Z, Liu J, et al. $\mathrm{MoS}_{2}$ nanosheets decorated with ultrafine $\mathrm{Co}_{3} \mathrm{O}_{4}$ nanoparticles for high-performance electrochemical capacitors. Electrochim Acta, 2015, 182: 376-382

76 Meng F, Fang Z, Li Z, et al. Porous $\mathrm{Co}_{3} \mathrm{O}_{4}$ materials prepared by solid-state thermolysis of a novel Co-MOF crystal and their superior energy storage performances for supercapacitors. J Mater Chem A, 2013, 1: 7235-7241

77 Maiti S, Pramanik A, Mahanty S. Extraordinarily high pseudocapacitance of metal organic framework derived nanostructured cerium oxide. Chem Commun, 2014, 50: 11717-11720

78 Liu K, You H, Jia G, et al. Coordination-induced formation of one-dimensional nanostructures of europium benzene-1,3,5-tricarboxylate and its solid-state thermal transformation. Cryst Growth Des, 2009, 9: 3519-3524

79 Chen S, Xue M, Li Y, et al. Rational design and synthesis of $\mathrm{Ni}_{x}$ $\mathrm{Co}_{3-x} \mathrm{O}_{4}$ nanoparticles derived from multivariate MOF-74 for supercapacitors. J Mater Chem A, 2015, 3: 20145-20152

80 Salunkhe RR, Tang J, Kamachi Y, et al. Asymmetric supercapacitors using 3D nanoporous carbon and cobalt oxide electrodes synthesized from a single metal-organic framework. ACS Nano, 2015, 9: 6288-6296

81 Liu S, Tong M, Liu G, et al. S,N-containing Co-MOF derived $\mathrm{Co}_{9} \mathrm{~S}_{8} @ \mathrm{~S}, \mathrm{~N}$-doped carbon materials as efficient oxygen electrocatalysts and supercapacitor electrode materials. Inorg Chem Front, 2017, 4: 491-498

82 Wang DW, Li F, Liu M, et al. 3D aperiodic hierarchical porous graphitic carbon material for high-rate electrochemical capacitive energy storage. Angew Chem Int Ed, 2008, 47: 373-376

83 Wang Q, Jiao L, Du H, et al. $\mathrm{Co}_{3} \mathrm{~S}_{4}$ hollow nanospheres grown on graphene as advanced electrode materials for supercapacitors. J Mater Chem, 2012, 22: 21387-21391

84 Wang Q, Jiao L, Du H, et al. $\mathrm{Fe}_{3} \mathrm{O}_{4}$ nanoparticles grown on graphene as advanced electrode materials for supercapacitors. J 
Power Sources, 2014, 245: 101-106

85 Liu S, Zhao Q, Tong M, et al. Ultrafine nickel-cobalt alloy nanoparticles incorporated into three-dimensional porous graphitic carbon as an electrode material for supercapacitors. J Mater Chem A, 2016, 4: 17080-17086

$86 \mathrm{Wu} \mathrm{Y}$, Liu S, Zhao K, et al. Chemical deposition of $\mathrm{MnO}_{2}$ nanosheets on graphene-carbon nanofiber paper as free-standing and flexible electrode for supercapacitors. Ionics, 2016, 22: 11851195

87 Zhang Z, Li X, Wang C, et al. Polyacrylonitrile and carbon nanofibers with controllable nanoporous structures by electrospinning. Macromol Mater Eng, 2009, 294: 673-678

88 Teng M, Qiao J, Li F, et al. Electrospun mesoporous carbon nanofibers produced from phenolic resin and their use in the adsorption of large dye molecules. Carbon, 2012, 50: 2877-2886

89 Zhao M, Wang Y, Ma Q, et al. Ultrathin 2D metal-organic framework nanosheets. Adv Mater, 2015, 27: 7372-7378

90 Meng W, Chen W, Zhao L, et al. Porous $\mathrm{Fe}_{3} \mathrm{O}_{4} /$ carbon composite electrode material prepared from metal-organic framework template and effect of temperature on its capacitance. Nano Energy, 2014, 8: 133-140

91 Salunkhe RR, Kamachi Y, Torad NL, et al. Fabrication of symmetric supercapacitors based on MOF-derived nanoporous carbons. J Mater Chem A, 2014, 2: 19848-19854

92 Cao F, Zhao M, Yu Y, et al. Synthesis of two-dimensional $\mathrm{CoS}_{1.097} /$ nitrogen-doped carbon nanocomposites using metal-organic framework nanosheets as precursors for supercapacitor application. J Am Chem Soc, 2016, 138: 6924-6927

93 Li GC, Liu PF, Liu R, et al. MOF-derived hierarchical doubleshelled $\mathrm{NiO} / \mathrm{ZnO}$ hollow spheres for high-performance supercapacitors. Dalton Trans, 2016, 45: 13311-13316

94 Yang J, Zheng C, Xiong P, et al. Zn-doped Ni-MOF material with a high supercapacitive performance. J Mater Chem A, 2014, 2: 19005-19010

95 Weng Q, Wang X, Wang X, et al. Supercapacitive energy storage performance of molybdenum disulfide nanosheets wrapped with microporous carbons. J Mater Chem A, 2015, 3: 3097-3102

96 Wang Z, Jia W, Jiang M, et al. Microwave-assisted synthesis of layer-by-layer ultra-large and thin NiAl-LDH/RGO nanocomposites and their excellent performance as electrodes. Sci China Mater, 2015, 58: 944-952

97 Mahmood N, Tahir M, Mahmood A, et al. Role of anions on structure and pseudocapacitive performance of metal double hydroxides decorated with nitrogen-doped graphene. Sci China Mater, 2015, 58: 114-125

98 Li X, Hao C, Tang B, et al. Supercapacitor electrode materials with hierarchically structured pores from carbonization of MWCNTs and ZIF-8 composites. Nanoscale, 2017, 9: 2178-2187

99 Xu X, Wang M, Liu Y, et al. In situ construction of carbon nanotubes/nitrogen-doped carbon polyhedra hybrids for supercapacitors. Energy Storage Mater, 2016, 5: 132-138

100 Wen P, Gong P, Sun J, et al. Design and synthesis of Ni-MOF/ CNT composites and $\mathrm{rGO} /$ carbon nitride composites for an asymmetric supercapacitor with high energy and power density. J Mater Chem A, 2015, 3: 13874-13883

101 Zhang Y, Lin B, Sun Y, et al. Carbon nanotubes@metal-organic frameworks as Mn-based symmetrical supercapacitor electrodes for enhanced charge storage. RSC Adv, 2015, 5: 58100-58106

102 Zhang Y, Lin B, Wang J, et al. All-solid-state asymmetric supercapacitors based on $\mathrm{ZnO}$ quantum dots/carbon/CNT and porous
$\mathrm{N}$-doped carbon/CNT electrodes derived from a single ZIF-8/ CNT template. J Mater Chem A, 2016, 4: 10282-10293

103 Kim D, Kim DW, Hong WG, et al. Graphene/ZIF-8 composites with tunable hierarchical porosity and electrical conductivity. J Mater Chem A, 2016, 4: 7710-7717

104 Zhou Y, Mao Z, Wang W, et al. In-situ fabrication of graphene oxide hybrid Ni-based metal-organic framework (Ni-MOFs@GO) with ultrahigh capacitance as electrochemical pseudocapacitor materials. ACS Appl Mater Interfaces, 2016, 8: 28904-28916

105 Yin D, Huang G, Sun Q, et al. $\mathrm{RGO} / \mathrm{Co}_{3} \mathrm{O}_{4}$ composites prepared using GO-MOFs as precursor for advanced lithium-ion batteries and supercapacitors electrodes. Electrochim Acta, 2016, 215: 410419

106 Wang L, Feng X, Ren L, et al. Flexible solid-state supercapacitor based on a metal-organic framework interwoven by electrochemically-deposited PANI. J Am Chem Soc, 2015, 137: 49204923

107 Lu C, Ben T, Xu S, et al. Electrochemical synthesis of a microporous conductive polymer based on a metal-organic framework thin film. Angew Chem Int Ed, 2014, 53: 6454-6458

108 Guo SN, Zhu Y, Yan YY, et al. (Metal-organic framework)polyaniline sandwich structure composites as novel hybrid electrode materials for high-performance supercapacitor. J Power Sources, 2016, 316: 176-182

109 Guo B, Yang Y, Hu Z, et al. Redox-active organic molecules functionalized nitrogen-doped porous carbon derived from metal-organic framework as electrode materials for supercapacitor. Electrochim Acta, 2017, 223: 74-84

110 Maiti S, Pramanik A, Mahanty S. Influence of imidazolium-based ionic liquid electrolytes on the performance of nano-structured $\mathrm{MnO}_{2}$ hollow spheres as electrochemical supercapacitor. RSC Adv, 2015, 5: 41617-41626

111 Jiang Z, Li Z, Qin Z, et al. LDH nanocages synthesized with MOF templates and their high performance as supercapacitors. Nanoscale, 2013, 5: 11770-11775

112 Srimuk P, Luanwuthi S, Krittayavathananon A, et al. Solid-type supercapacitor of reduced graphene oxide-metal organic framework composite coated on carbon fiber paper. Electrochim Acta, 2015, 157: 69-77

113 Fu D, Li H, Zhang XM, et al. Flexible solid-state supercapacitor fabricated by metal-organic framework/graphene oxide hybrid interconnected with PEDOT. Mater Chem Phys, 2016, 179: 166173

114 Banerjee PC, Lobo DE, Middag R, et al. Electrochemical capacitance of Ni-doped metal organic framework and reduced graphene oxide composites: more than the sum of its parts. ACS Appl Mater Interfaces, 2015, 7: 3655-3664

Acknowledgements This work was supported by the Fundamental Research Funds for Central Universities' through Beihang University and the Queensland Government through the Q-CAS Collaborative Science Fund 2016 "Graphene-Based Thin Film Supercapacitors".

Author contributions Zhao Y wrote the manuscript. Liu J organized the manuscript structure and contributed to the general discussion; Horn $\mathrm{M}$ and Motta $\mathrm{N}$ revised the manuscript. Hu M and Li Y contributed to the general contribution.

Conflict of interest The authors declare that they have no conflict interest. 


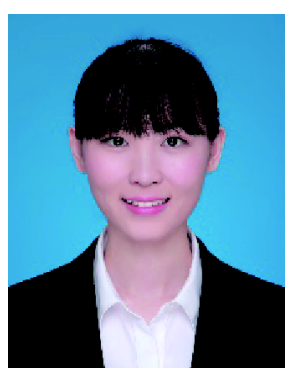

Yujie Zhao received her Master's degree from Chongqing University, China, in 2017. Currently, she is a PhD student at Beihang University, China. Her research interest is focused on functional nanomaterials and related energy applications.

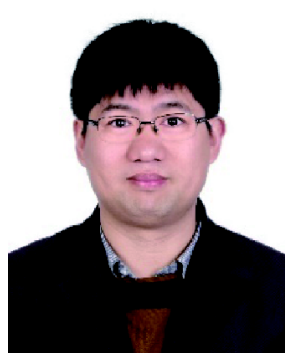

Jinzhang Liu received his PhD degree in Condensed Matter Physics from Lanzhou University in 2006. He then continued his academic career in South Korea (2006-2011) and Australia (2011-2015). Currently, he is an associated professor at the School of Materials Science and Engineering of Beihang University. His research interests include the synthesis of carbon materials and their applications in supercapacitors and solar-powered desalination.

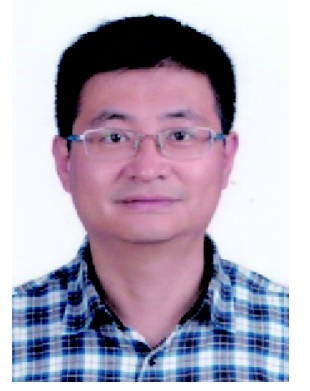

Yan Li is a professor of the School of Materials Science and Engineering of Beihang University. He received his PhD degree form Dalian University of Technology in 2001. His current research interests include shape memory materials, biomedical materials, supercapacitors and battery materials.

\section{金属有机框架材料在超级电容器中的应用研究进展}

赵昱颉 ${ }^{1}$, 刘金章 ${ }^{1^{*}}$, Michael Horn ${ }^{2}$, Nunzio Motta ${ }^{2}$, 胡明俊 ${ }^{1}$, 李岩 ${ }^{*}$

摘要 金属有机骨架材料具有结构多样、孔径可调、比表面积大及化学反应活性位点丰富等优点, 因此有望成为广泛应用于电化学储能 器件的电极材料, 如锂离子电池、燃料电池及超级电容器等. 近十年来金属有机骨架材料及其衍生物材料引起了广泛的研究与应用. 同 时, 金属有机骨架材料及其衍生的纳米材料作为电化学储能器件中的关键电极也受到了广泛的研究. 本文主要综述了近期金属有机骨架 材料及其衍生物在超级电容器中的研究进展, 讨论了金属有机骨架材料制备超级电容器电极材料的方法及其在电化学储能领域体现出的 优异性能及特殊性质, 同时也总结了近期金属有机骨架材料的衍生物如碳材料、金属材料及复合物在超级电容器中的研究进展, 结果说 明金属有机骨架材料在发展新一代超级电容器电极材料领域具有重要的作用. 最后讨论了金属有机骨架材料及其衍生物目前在超级电容 器领域存在的问题以及未来可能的发展方向. 